\title{
Impact of gridpoint statistical interpolation scheme over Indian region
}

\author{
SURYA K DUTTA* and V S PRASAD** \\ National Centre for Medium Range Weather Forecasting, Ministry of Earth Sciences, \\ A-50, Sector 62, Noida 201 307, India. \\ *Corresponding author.e-mail: surya@ncmrwf.gov.in \\ **e-mail: vsprasad@ncmrwf.gov.in
}

\begin{abstract}
An analysis system experiment was conducted for the month of June 2008 with Gridpoint Statistical Interpolation (GSI) analysis scheme using NCMRWF's (National Centre for Medium Range Weather Forecasting) T254L64 model. Global analyses were carried out for all days of the month and respective forecast runs are made up to 120-hr. These analyses and forecasts are inter-compared with the operational T254L64 model outputs which uses Spectral Statistical Interpolation (SSI) analysis scheme. The prime objective of this study is to assess the impact of GSI analysis scheme with special emphasis on Indian summer monsoon as compared to SSI.

GSI analysis scheme do have positive impact over India and its surrounding regions. Though not for all but for some fields it is in edge over Spectral Statistical Analysis Scheme. Patterns for the forecast mean error; anomaly correlation and $\mathrm{S}_{1}$ scores with respect to the respective analyses are same for both GSI and SSI. Both have increasing $\mathrm{S}_{1}$ scores, decreasing mean errors and anomaly correlation with the advance of forecast days. The vector wind RMSE of the model forecasts with respect to the analyses is lower for GSI at $850 \mathrm{hPa}$ and higher at $250 \mathrm{hPa}$. But over tropics GSI is better at both levels. The temperature field of GSI has higher correlation and lower RMSE at both 850 and $250 \mathrm{hPa}$ pressure levels. There are improvements in systematic errors for 850 and $200 \mathrm{hPa}$ temperature field in GSI compared to that in SSI. The depression centre in GSI analysis is closer to observation but has produced more intense depression compared to that of SSI. Rainfall forecast of SSI is better at day-1 whereas GSI is closer to the observation at day- 5 forecasts valid at the same day.
\end{abstract}

\section{Introduction}

A Global Data Assimilation and Forecasting (GDAF) system for providing medium range weather forecasts over Indian region is operational at National Centre for Medium Range Weather Forecasting (NCMRWF), India since 1994. Its analysis system was based on global Spectral Statistical Interpolation (SSI) scheme (Parrish and Derber 1992), till January 2009 when Gridpoint Statistical Interpolation (GSI) analysis scheme became operational. In SSI analysis system, the spectral coefficients used in the spectral model are analyzed directly using the same basic equations as statistical (optimal) interpolation. The analysis variables in SSI are spectral coefficients instead of grid point values. It uses all observations at once to solve a single global problem (Parrish and Derber 1992). In this formulation, the background error covariances are computed with the assumption that uncorrelated errors in spectral space leads to homogeneous, isotropic statistics in grid space. This is a major weakness of the system and thus there is a need to develop new formulation to allow both the variance and correlation length scale to vary nontrivially in all space directions.

Keywords. Atmospheric sciences; modelling; monsoons. 
GSI analysis scheme (NCEP's current operational analysis system) (Wu et al 2002) is the evolutionary combination of the global SSI analysis system and the regional ETA 3D-VAR. It replaces spectral definition for background errors with grid point (physical space) version based on recursive filters. This global 3D-VAR in physical space is as effective as 3D-VAR in spectral space with latitude-dependent structure functions and other error statistics. Diagonal background error covariance in spectral space (in SSI) allows little control over the spatial variation of the error statistics as the structure function is limited to being geographically homogeneous and isotropic about its center (Parrish and Derber 1992; Courtier et al 1998). GSI allows greater flexibility in terms of inhomogeneity and anisotropy for background error statistics (Wu et al 2002). Thus major improvement of GSI over SSI analysis scheme is its latitude-dependent structure functions and has more appropriate background errors in the tropics. The background error covariances are isotropic and homogenous in the zonal direction.

$\mathrm{Wu}$ et al (2002) tested the proposed analysis system against operational SSI at NCEP by conducting a two low resolution T62 data assimilation experiments. They reported that GSI had a small impact on extra-tropics but it had shown consistent positive impact in tropics. It proves that the newly gained freedom in the background error's spatial variation is beneficial to forecasts compared with that of the SSI in which the statistics represent the global characteristics lacking any spatial modulation. To gain these benefits NCEP replaced SSI analysis system with GSI based system.

Southwest monsoon season is a very important season for India as major part of the annual rainfall of this region occurs during this part of the year. Further, during this period circulation characteristics of the atmosphere and ocean of the tropical Indian Ocean region are known to be quite different from the rest of tropics. As such the impact of any kind of data assimilation methods may differ from the rest of regions. Taking these facts into account, in this study a detailed examination of the GSI analysis system is conducted for the month of June 2008 and the results are compared with that of SSI system operational in NCMRWF. The paper is arranged as follows. Section 2 gives a brief description of experimental setup, SSI and GSI formulation. Results are discussed in section 3 and they are subsequently summarized in section 4 .

\section{Experimental setup}

The present NCMRWF's GDAF system is based on NCEP GFS (Global Forecast System). The analysis and forecasts are carried out at triangular truncation of wave number T254 and with 64 levels in vertical. Global analyses are carried out four times $(00 z, 06 z, 12 z$ and $18 z)$ a day with intermittent time scheme and with all the observations that are received at NCMRWF in \pm 3 hours time window. Model forecasts are carried up to 120 hours. The details of the implementation are documented by Rajagopal et al (2007). The observations used in the assimilation system are tabulated in table 1. In order to assess the impact of new analysis system, global analysis-forecast are repeated for the month of June 2008 with GSI analysis scheme. Global analysis was done for all days of the whole month and 120-hr model forecasts are made for all the days. The analyses and forecasts are inter-compared with the NCMRWF's operational T254L64 model outputs which uses SSI analysis scheme. The input observational dataset (table 1) used is the same for both analyses systems. The prime objective of this study is to determine the impact of GSI analysis scheme over India and its surrounding regions. The results from GSI experiments are compared with that of SSI to assess its improvement.

\subsection{SSI formulation}

SSI analysis system is closely related to a threedimensional (3D) variational analysis system developed at the European Centre for Medium Range Weather Forecasts (ECMWF) (Pailleux 1990). The objective function minimized by SSI is given by:

$$
J=\frac{1}{2}\left[x^{T} B^{-1} x+(L x-y)^{T}(F+O)^{-1}(L x-y)\right]
$$

where $x$ is an $N$-component vector of analysis increments; $B$ is the $N \times N$ forecast-error covariance matrix; $O$ is the $M \times M$ observational error covariance matrix; $F$ is the $M \times M$ representativeness error covariance matrix; $L$ is a linear transformation operator that converts the analysis variables to the observation type and location. $y$ is an $M$-component vector of observational residuals; i.e., $y=y_{\text {obs }}-L x_{\text {guess }} ; N$ is the number of degrees of freedom in the analysis; and $M$ is the number of observations.

The analysis variables in SSI defined in spectral space are closely related to the sigma-coordinate coefficients of the spherical harmonic expansions of vorticity, divergence, temperature, logarithm of surface pressure and mixing ratio. As the analysis variables are spectral, the forecast error covariance is defined in terms of the aforesaid spectral variables. In defining the analysis variables, the balanced components of the mass and momentum fields have been combined into a single variable. 
Table 1. Observations currently used in NCMRWF's assimilation system.

\begin{tabular}{ll}
\hline Observation type & Variables \\
\hline Radiosonde & $U, V, T, q, P_{s}$ \\
Pibal winds & $U, V$ \\
Wind profilers & $U, V$ \\
Surface land observations & $P_{s}$ \\
Surface ship and buoy observations & $U, V, T, q, P_{s}$ \\
Conventional aircraft observations (AIREP) & $U, V, T$ \\
AMDAR aircraft observations & $U, V, T$ \\
ACARS Aircraft observations & $U, V, T$ \\
GMS/MTSAT AMV (BUFR) & $U, V, T$ \\
INSAT AMV (SATOB) & $U, V, T$ \\
METEOSAT AMV (BUFR) & $U, V, T$ \\
GOES (BUFR) & $U, V, T$ \\
SSM/I & Surface wind speed \\
Scatterometer (QSCAT) & $10 \mathrm{~m} U, V$ \\
AMSU-A radiance & Brightness temperature \\
AMSU-B radiance & Brightness temperature \\
HIRS radiance & Brightness temperature \\
SBUV ozone & Total ozone \\
\hline
\end{tabular}

This, in turn allows the balance between the mass and momentum fields to be implicitly included. Unlike GSI whose background error statistics defined in grid space is latitude dependent, the background error statistics of SSI defined in spectral space are geographically homogeneous. The wind error variance is homogeneous over the globe, but the balanced part of the mass errors becomes very small as the equator is approached.

\subsection{GSI formulation}

GSI analysis scheme is a global 3D-VAR scheme in physical space. The cost function that it minimizes is

$$
J=\frac{1}{2}\left[x^{T} B^{-1} x+(H x-y)^{T} R^{-1}(H x-y)\right]
$$

where $x$ is the vector of analysis increment, $B$ is the background error covariance matrix, $y$ is the vector of the observational residuals, i.e., $y=$ $y_{\text {obs }}-H x_{\text {guess }}, R$ is the observational and representativeness error covariance matrix, and $H$ is the transformation operator from the analysis variable to the form of the observational vector.

The analysis variables unlike SSI that are defined on the grid are streamfunction $(\psi)$; unbalanced part of velocity potential $(X)$; the unbalanced part of surface pressure $(P)$; pseudo-relative humidity $(q)$ (defined as water vapour mixing ratio divided by the saturated value from the guess field). In GSI, the background error co-variance matrix is constructed in physical space using recursive filters. The recursive filters used are self-adjoint and have the ability to accommodate geographically adaptive horizontal scales. They also have good amplitude control and so are able to apply the estimated background error variances precisely. These filters have boundary treatments that avoid any serious numerical artifacts so that they can be applied to many sub-domains in a way that allows the constituent parts to be merged consistently back together. In GSI analysis scheme, the globe is divided into three pieces: two Cartesian polar patches and a zonal band in between. The recursive filters are applied to each sub-domain.

\section{Results and discussions}

In the present analysis experiment, detailed study has been conducted over Northern and Southern Hemispheres, Tropics $\left(30^{\circ} \mathrm{S}-30^{\circ} \mathrm{N}\right)$ and Indian region $\left(10^{\circ} \mathrm{S}-40^{\circ} \mathrm{N}, 40^{\circ}-100^{\circ} \mathrm{E}\right)$. The impact over Northern and Southern Hemispheres are in agreement to that obtained by Wu et al (2002). GSI is seen to cast more impact over Indian region and Tropics than that over the two hemispheres. Special emphasis is given over the Indian summer monsoon and the discussions so made are mainly for Indian region. The discussions have been grouped into forecast $v s$. analyses, forecast vs. observation and synoptic study. All the scores computed are averaged over the respective region in space and time. 


\subsection{Forecast vs. analyses}

Performance of the model forecasts in terms of their respective analyses is investigated first. For the forecast vs. analyses study, comparisons were made in terms of mean error, correlation coefficient and $\mathrm{S}_{1}$ scores. Mean error is the difference of the forecasts from their respective analyses averaged over the region and time. The correlation coefficient is the correlation between the observed and forecast anomalies. $\mathrm{S}_{1}$ scores represent how well the forecast gradients correspond to the observed gradients. It is represented as:

$$
S_{1}=100 \cdot \frac{\sum_{i=1}^{n}\left(e_{g}\right)_{i} \cos \varphi_{i}}{\sum_{i=1}^{n}\left(G_{L}\right)_{i} \cos \varphi_{i}}
$$

where,

$$
\begin{aligned}
e_{g} & =\left\{\left|\frac{\partial}{\partial x}\left(x_{f}-x_{v}\right)\right|+\left|\frac{\partial}{\partial y}\left(x_{f}-x_{v}\right)\right|\right\} \\
G_{L} & =\max \left[\left|\frac{\partial_{x_{f}}}{\partial_{x}}\right| \cdot\left|\frac{\partial_{x_{v}}}{\partial_{x}}\right|\right]+\max \left[\left|\frac{\partial_{x_{f}}}{\partial_{y}}\right| \cdot\left|\frac{\partial_{x_{v}}}{\partial_{y}}\right|\right]
\end{aligned}
$$

where, $x_{\mathrm{f}}$ is the forecast value of the parameter in question; $x_{\mathrm{v}}$ is the corresponding verifying value (analyzed); $n$ is the number of grid points in the verification area; and $\cos \varphi_{\mathrm{i}}$ is cosine of latitude of grid point $i$.
$S_{1}$ score ranges from 0 to $\infty$. Perfect $S_{1}$ score is zero. It is usually applied to geopotential height or sea level pressure fields in meteorology. Because $S_{1}$ depends only on gradients, good scores can be achieved even when the forecast values are biased. For the present study, $\mathrm{S}_{1}$ scores were computed for the geopotential height. Mean error of the simulated geopotential height at $850 \mathrm{hPa}$ and $250 \mathrm{hPa}$ is tabulated in table 2 and $S_{1}$ scores are tabulated in table 3. Patterns for forecast mean error and $S_{1}$ scores are same for both GSI and SSI over Indian region. With the forecast days, both the simulations show increasing $S_{1}$ scores and decreasing mean error. GSI analysis scheme shows lower magnitude of mean errors than SSI analysis scheme.

Here at first, performance of both GSI and SSI over tropics is investigated in greater detail. Forecast from both GSI and SSI schemes have been compared with the analyses obtained from the two schemes over the tropics. Upper parts in figure 1 depict the mean anomaly correlation over tropics averaged for the study period for the forecast days. The positive impact of GSI with consistent higher values over the lower layers (figure 1a and b) for both wind and temperature is clearly visible. Also for upper layer (figure 1c and d), GSI temperature correlation is higher. The results are mixed for wind correlation at upper layer. The lower parts in figure 1 give the difference of anomaly

\begin{tabular}{|c|c|c|c|c|}
\hline \multirow[b]{3}{*}{ Days } & \multicolumn{4}{|c|}{ Indian Region } \\
\hline & \multicolumn{2}{|c|}{$850 \mathrm{hPa}$} & \multicolumn{2}{|c|}{$250 \mathrm{hPa}$} \\
\hline & GSI & SSI & GSI & SSI \\
\hline 1 & -8.1 & -12.2 & -4.5 & -7.4 \\
\hline 2 & -9.6 & -14.6 & -7.4 & -9.7 \\
\hline 3 & -7.5 & -14.1 & -5.3 & -9.9 \\
\hline 4 & -7.2 & -14 & -6.5 & -9.6 \\
\hline 5 & -7.8 & -13.6 & -8.4 & -9.4 \\
\hline
\end{tabular}

Table 2. Mean error for GSI and SSI geopotential height at 850 and $250 h P a$.

Table 3. $S_{1}$ score for GSI and SSI geopotential height at 850 and $250 h P a$.

\begin{tabular}{lllll}
\hline & \multicolumn{4}{c}{ Indian Region } \\
\cline { 2 - 5 } Days & GSI & SSI & GSI & SSI \\
\cline { 2 - 5 } & 35.2 & 29.1 & 35.1 & 23.9 \\
1 & 38.2 & 33.8 & 37.8 & 27.3 \\
2 & 41.9 & 37.6 & 40.2 & 30.3 \\
4 & 45.1 & 41.4 & 42.4 & 33.2 \\
5 & 47.4 & 44.6 & 44.3 & 36.3 \\
\hline
\end{tabular}


(a) AC: T P850 G2/TRO 00Z, 20080608-20080630

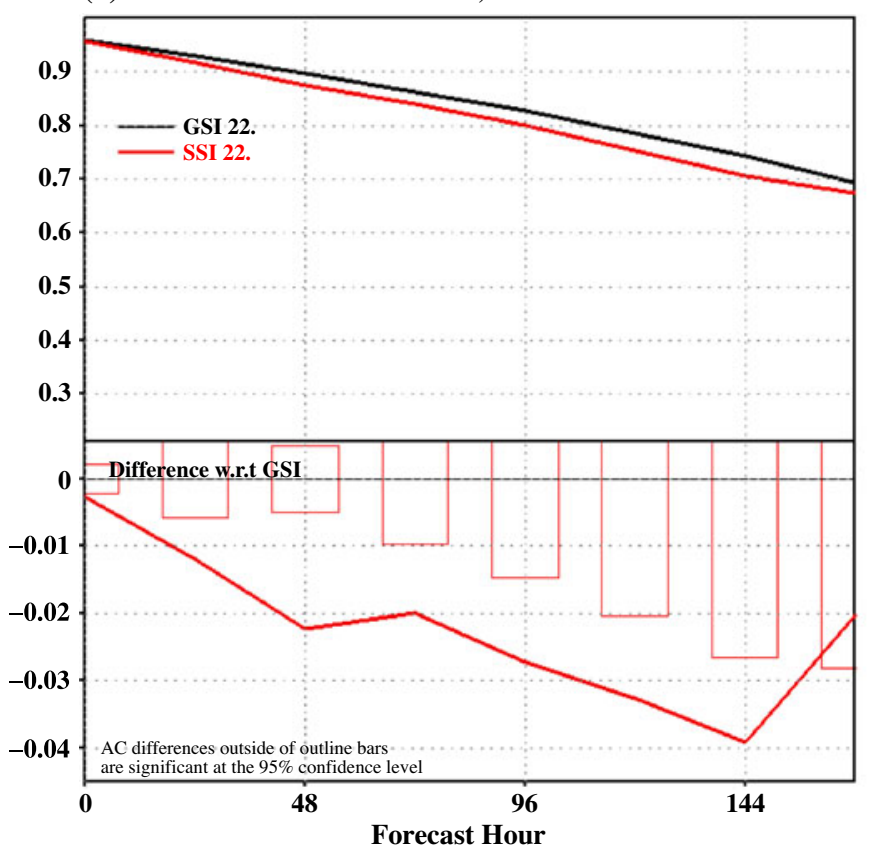

(c) AC: T P500 G2/TRO 00Z, 20080608-20080630

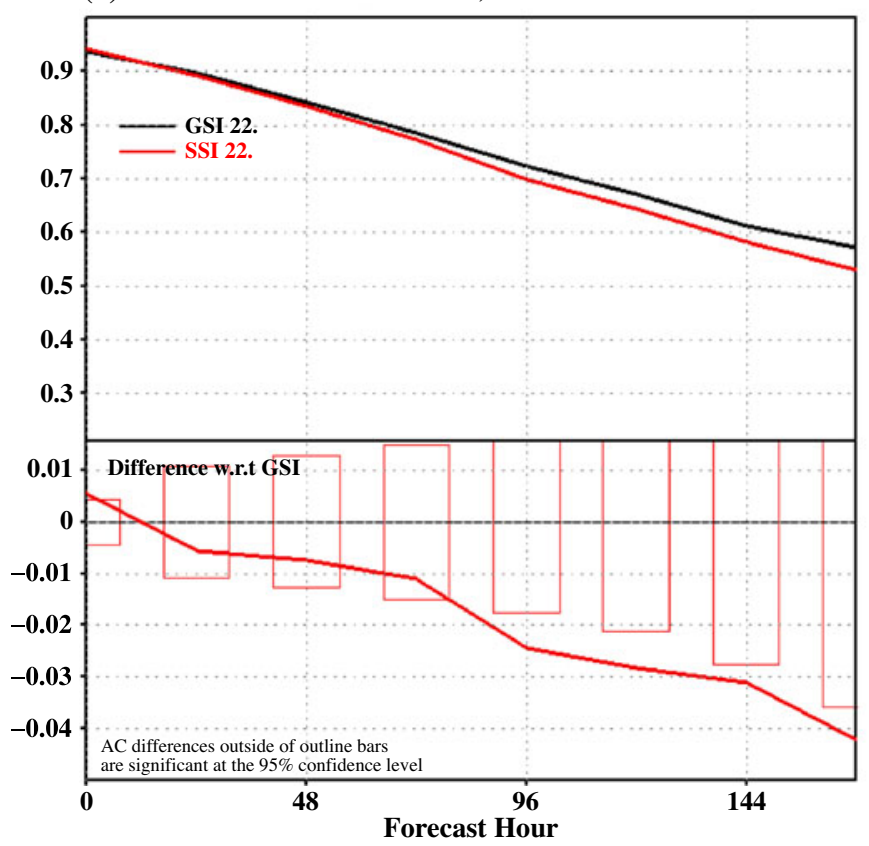

(b) AC: WIND P850 G2/TRO 00Z, 20080608-20080630

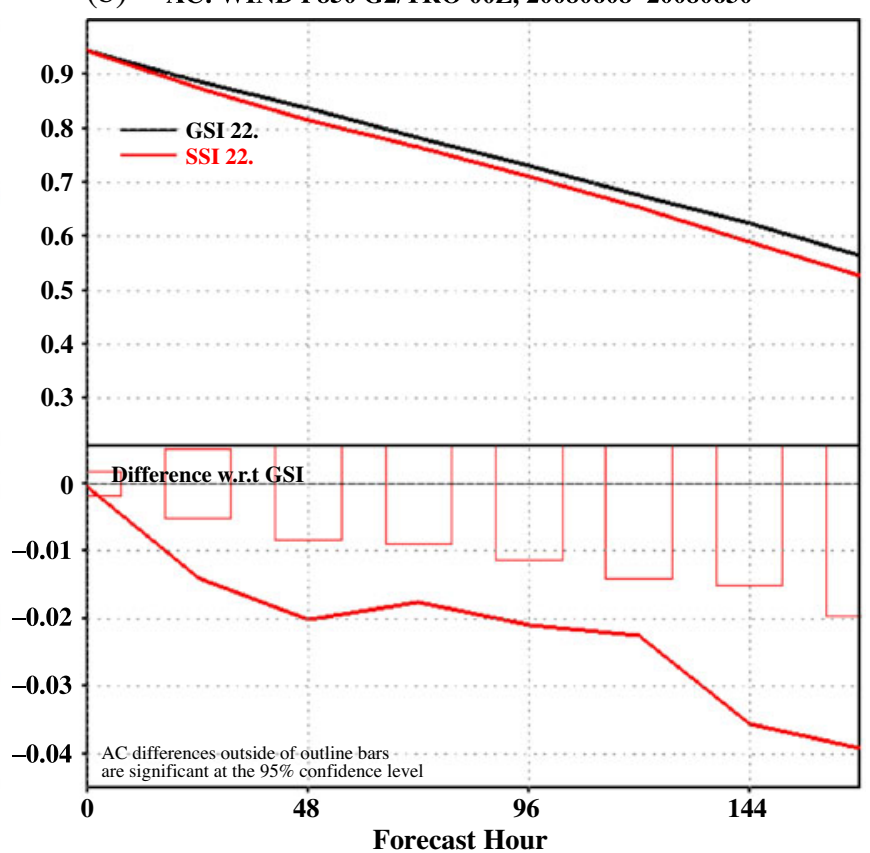

(d) AC: WIND P500 G2/TRO 00Z, 20080608-20080630

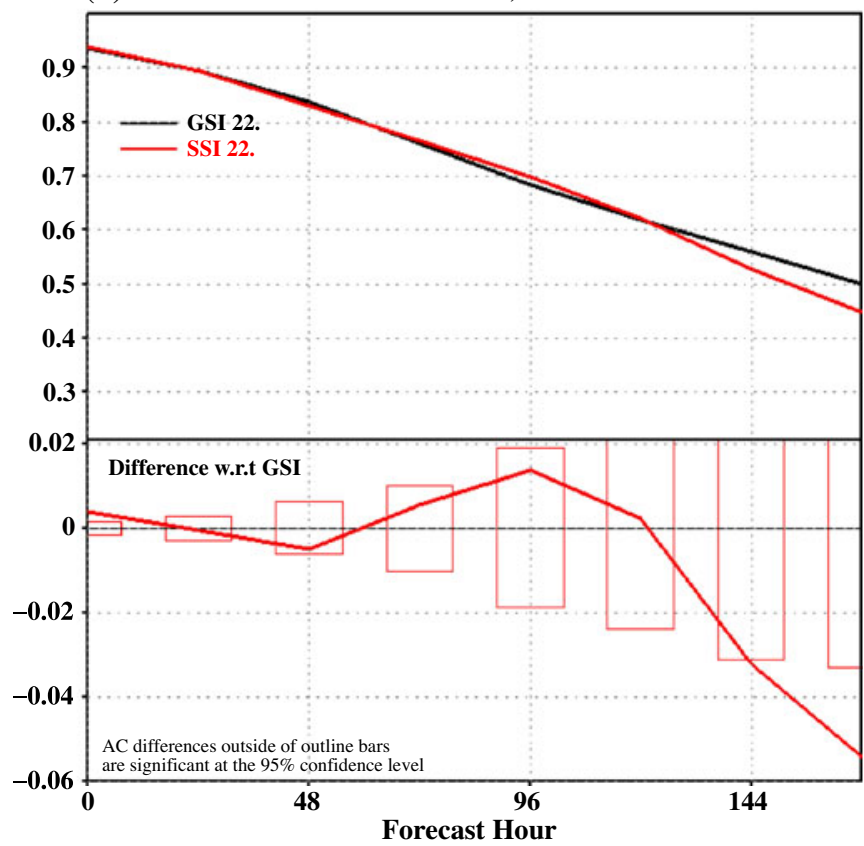

Figure 1. Anomaly correlation over tropics for day-1 to day-7 (upper part of the figures) and anomaly correlation difference of SSI w.r.t GSI along with their statistical significance (lower part of the figures) for temperature at (a) $850 \mathrm{hPa}$ and (c) $500 \mathrm{hPa}$, and for wind at (b) $850 \mathrm{hPa}$ and (d) $500 \mathrm{hPa}$.

correlation between GSI and SSI and their statistical significance. The difference values outside the histograms are significant at 95\% level of confidence. At $850 \mathrm{hPa}$, the differences are significant for all the forecast days for wind and from day- 1 to day- 6 for temperature. The differences are increasing with the forecast days. For the upper layer at $500 \mathrm{hPa}$, differences in temperature correlation are significant from day-4 to day-7. For wind, differences are significant only for the forecast valid at day-7. Figure 2 is arranged similar to figure 1 but depicting pattern correlation for GSI and SSI over tropics averaged over the study period. The features presented by pattern correlation entirely match that with those given by anomaly correlation. Both anomaly and pattern correlation for geopotential height (figure not included) also show consistent positive impact of GSI over tropics for 
T: Pattern Correlation

(a) P850 G2/TRO 00Z. 20080608-20080630 Mean

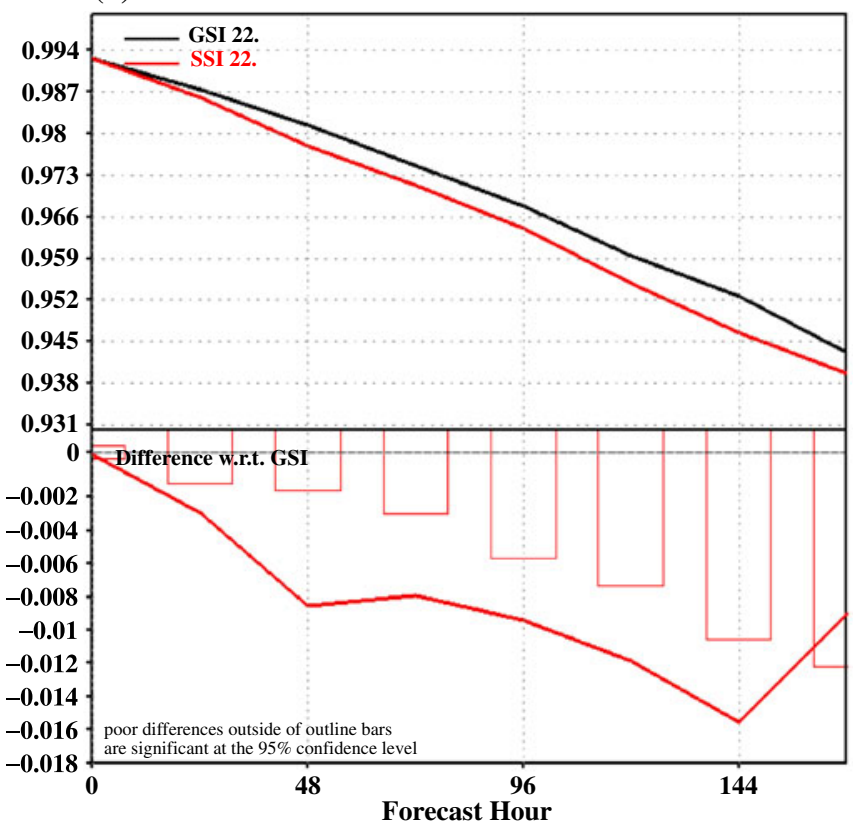

T: Pattern Correlation

(c) P800 G2/TRO 00Z. 20080608-20080630 Mean

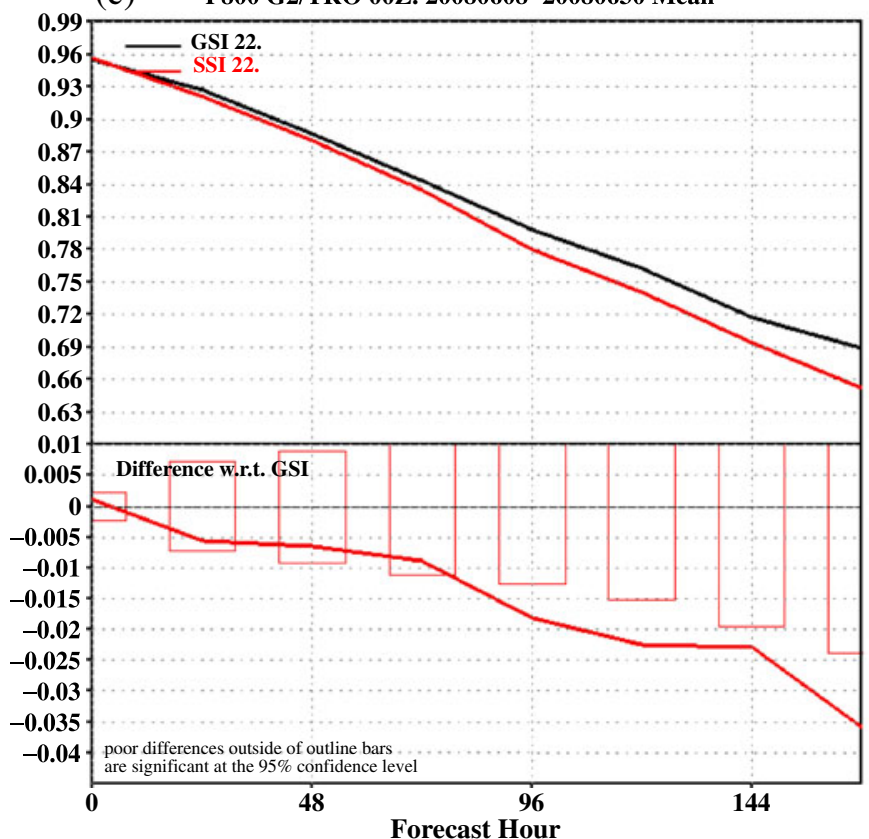

WIND: Pattern Correlation

(b) P850 G2/TRO 00Z. 20080608-20080630 Mean

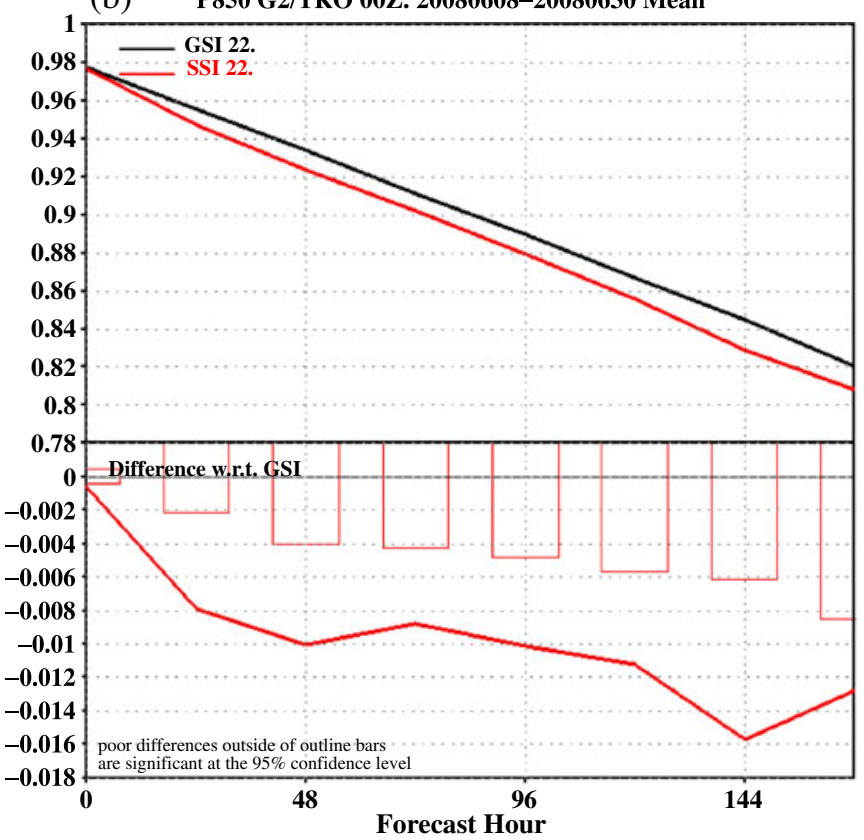

WIND: Pattern Correlation

(d) P800 G2/TRO 00Z. 20080608-20080630 Mean

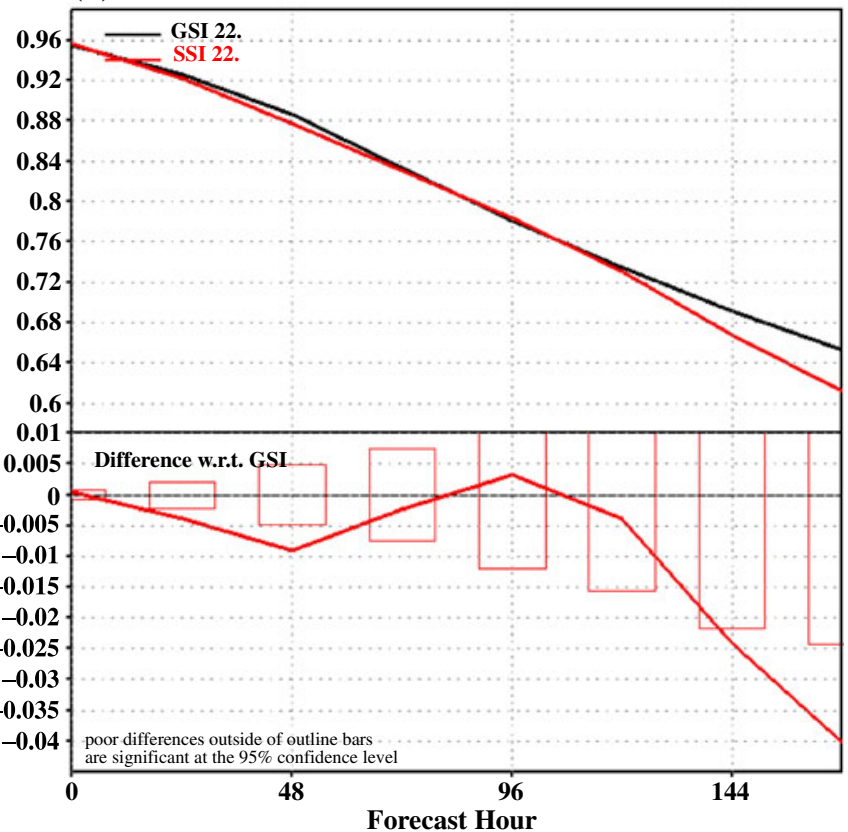

Figure 2. Same as figure 1 but presenting pattern correlation.

the lower layers. Over the upper layers the results are mixed. Indian region being a part of the tropics, impact of the analyses schemes over tropics will also be the same and highlighted over the Indian region. Here, only 500 hpa results are being shown as upper layer. The results are same for layers higher than $500 \mathrm{hPa}$.

Figure 3 shows the anomaly correlation coefficient for geopotential height and temperature (figure $3 \mathrm{a}$ and $\mathrm{b}$ ) and forecast RMSE for the same (figure $3 \mathrm{c}$ and $\mathrm{d}$ ) with respect to the respective analyses. It is seen from the figure that the anomaly correlation of the geopotential height for GSI is mostly higher than SSI at $850 \mathrm{hPa}$ over Indian region. The situation reverses at $250 \mathrm{hPa}$. For the temperature field, GSI has higher correlation and lower RMSE both at 850 and $250 \mathrm{hPa}$ (figure 3). Also, the vector wind RMSE is lower for GSI at 

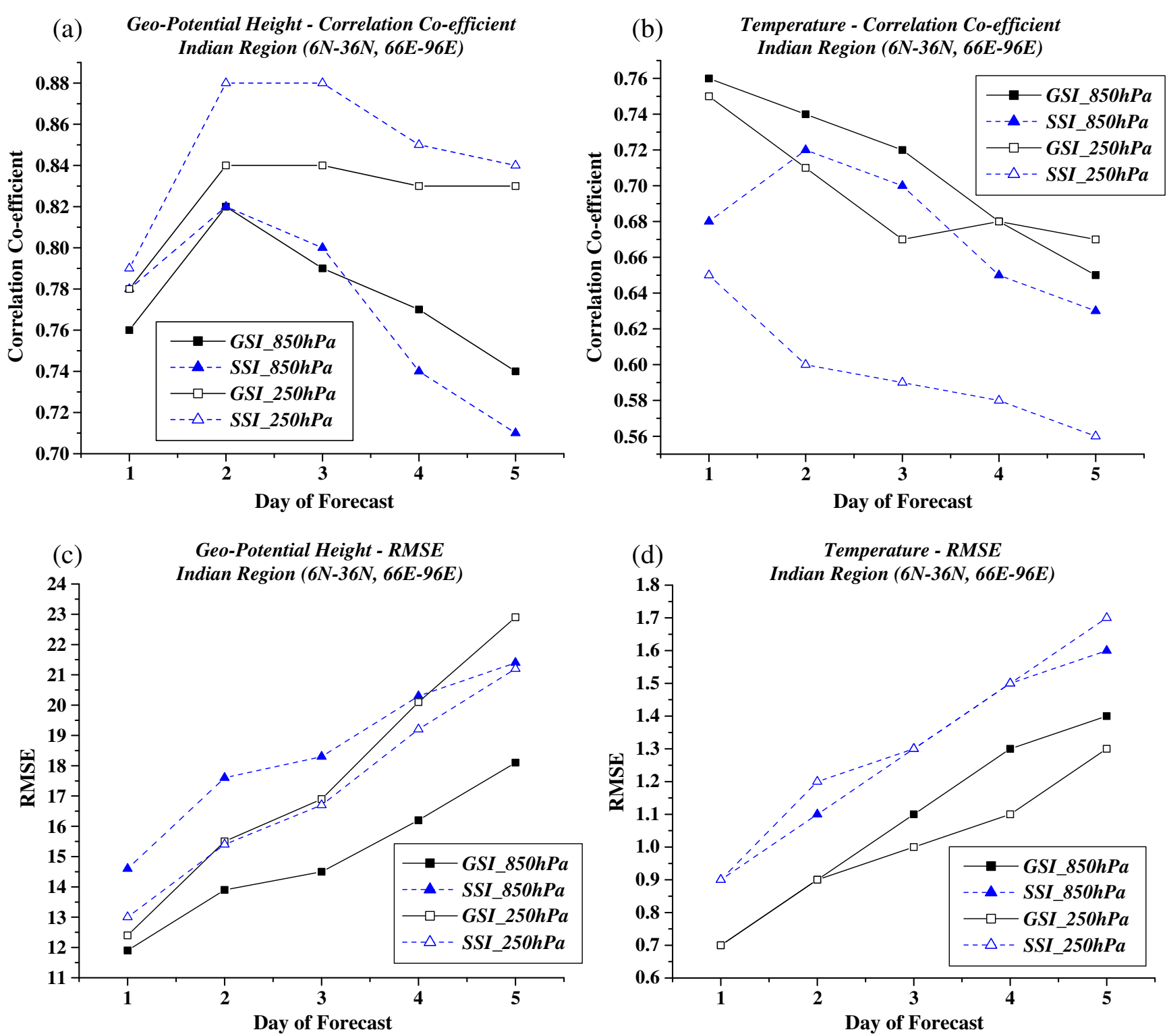

Figure 3. Anomaly correlation for (a) geopotential height and (b) temperature and forecast RMSE with respect to the respective analyses for (c) geopotential height (gpm) and (d) temperature (kelvin) over Indian region.

$850 \mathrm{hPa}$ and higher at $250 \mathrm{hPa}$ over the Indian region (figure not shown). But over tropics GSI is better at both the levels. However, SSI over the Indian region has lesser $S_{1}$ score than GSI. Only, skill score for 850 and $250 \mathrm{hPa}$ geopotential heights over Indian region is shown in table 3. It is seen that the $S_{1}$ score and its difference in between the two analyses scheme has lower magnitude over Northern and Southern Hemispheres (not included). This magnitude increases over tropics and the Indian region.

\subsection{Forecast vs. observation}

Impact over the model forecast using the analyses from the two different systems is also verified with respect to the radiosonde observations. For comparison of forecast vs. observation, forecast wind vector and temperature RMSE are computed at 850 and $250 \mathrm{hPa}$ pressure levels. Figure 4 presents the bar diagram plots for wind vector RMSE (figure 4a and b) and temperature RMSE (figure 4c and $\mathrm{d}$ ), respectively. Forecasts using GSI analyses is found to have lower vector wind RMSE at $850 \mathrm{hPa}$ at $3 \mathrm{rd}$ and 4 th day of forecasts. Though there is no improvement in $850 \mathrm{hPa}$ temperature RMSE using GSI, RMSE of $250 \mathrm{hPa}$ temperature forecasts of GSI is lower compared to that of SSI. Also, the forecasts using SSI analysis has lower zonal mean error of $\mathrm{u}$-wind and temperature at 850 and $250 \mathrm{hPa}$ pressure levels (figure not included). 

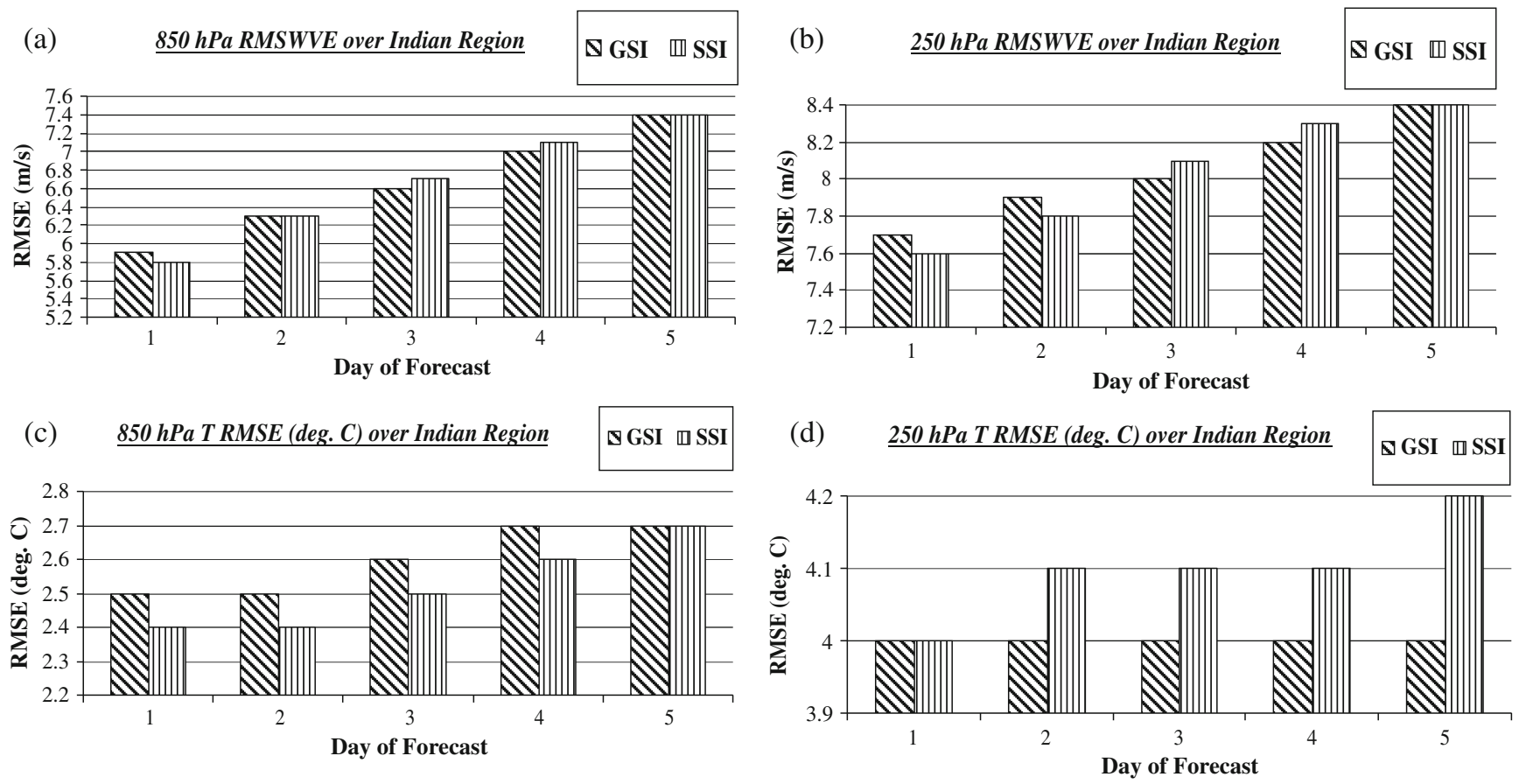

Figure 4. Vector wind RMSE at (a) $850 \mathrm{hPa}$ and (b) $250 \mathrm{hPa}$ pressure levels, and temperature $\mathrm{RMSE}\left({ }^{\circ} \mathrm{C}\right)$ at $(\mathbf{c}) 850 \mathrm{hPa}$ and (d) $250 \mathrm{hPa}$ pressure levels over Indian region for T254L64 forecasts with respect to observations using GSI and SSI analyses.

\subsection{Systematic errors}

Errors in the model forecasts may be due to the presence of systematic errors or due to the chaotic growth of errors in the initial conditions or due to both. Systematic errors of a numerical weather prediction model are basically the errors due to the assumptions taken for solving the model equations. The systematic errors mentioned here are computed by taking the difference of analyses and forecasts valid at the same day and averaged for the entire month of June 2008. Here for better understanding and comparison, the difference plot of the systematic errors of GSI and SSI for temperature, zonal and meridional wind at 850 and $200 \mathrm{hPa}$ are presented. Systematic error difference at day-1, day- 3 and day -5 forecasts are being shown. Figure 5 depicts the difference between the systematic errors in temperature forecast of GSI and SSI at 850 and $200 \mathrm{hPa}$ pressure levels. The negative regions have shadings of red and the positive regions are shaded with blue colour. Red shaded areas shows the regions where SSI is having greater systematic errors whereas blue shaded regions show areas where error in GSI is higher. The white regions are those areas where the difference is either zero or very less. All the parts of figure 5 are dominant with either white or red colour, especially over Indian landmass at $200 \mathrm{hPa}$. Figure 6 depicts the systematic error difference between GSI and SSI for zonal and meridional wind at $850 \mathrm{hPa}$.
Figure 7 is arranged similar to figure 6 but for $200 \mathrm{hPa}$ pressure level. Over India and surrounding regions, GSI has lower error in temperature forecast over both upper and lower layers. For both zonal and meridional wind, lower error in SSI is dominant. Improvement in systematic errors by GSI is mostly found over the peninsular and southern coast of India.

\subsection{Synoptic case study}

\subsubsection{Monsoon in June 2008}

Month of June is the month of monsoon onset over most of the Indian region. The onset dates vary from year to year. The monsoon winds approaches the west coast of India from a westerly or southwesterly direction. The normal winds at the higher levels over Indian region in the month of June are mostly easterly. Along the monsoon trough which is extensive regions of low pressure, strong rising motion is observed. This generalized flow pattern over the Indian region is verified through the latitudinal cross-section of zonal, meridional and vertical winds. In the year 2008, monsoon covered almost entire country except parts of west Rajasthan till 30th June. Rainfall activity over the country as a whole was above normal during the month. Central and northern parts of the country received excess rainfall. Among the chief synoptic features 

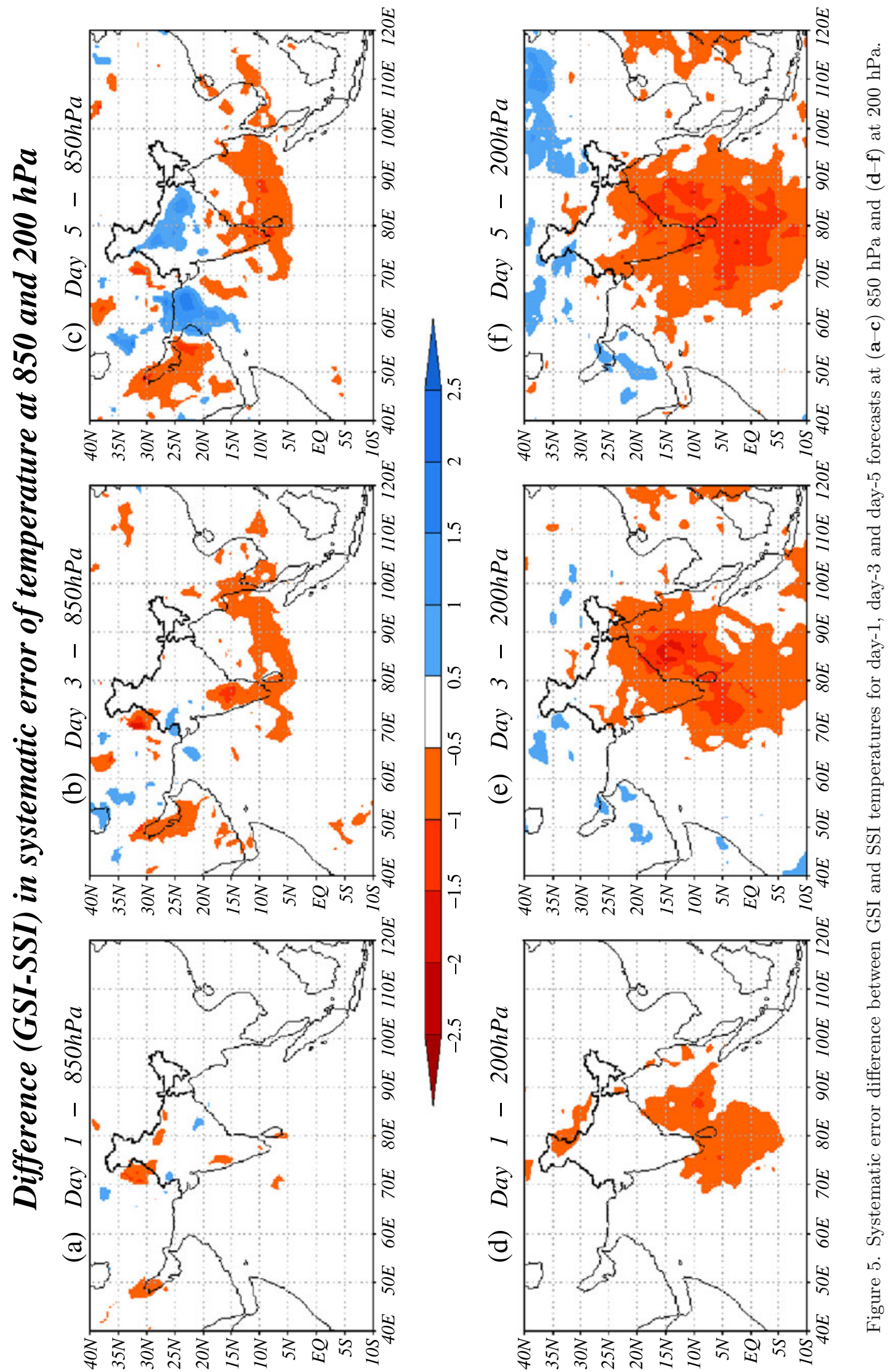

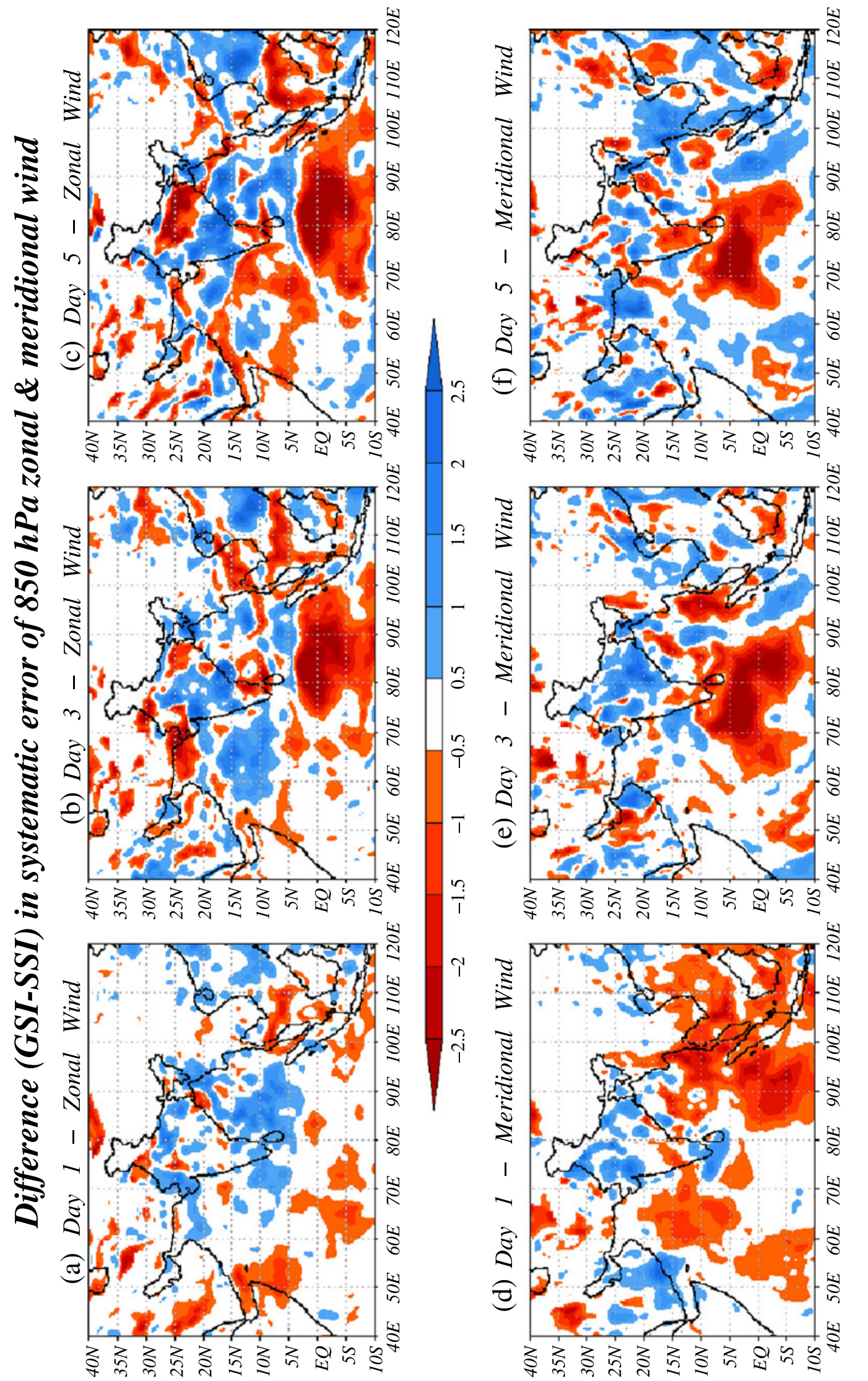

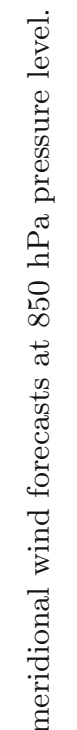
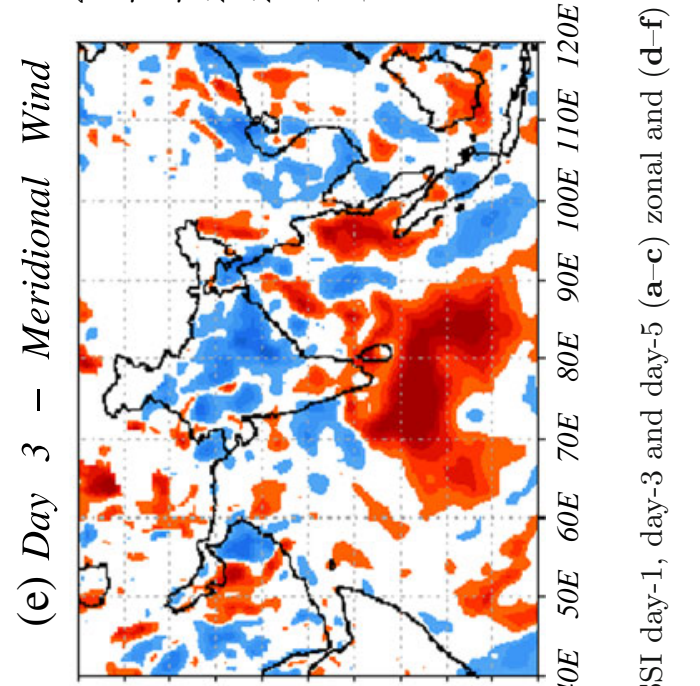

?
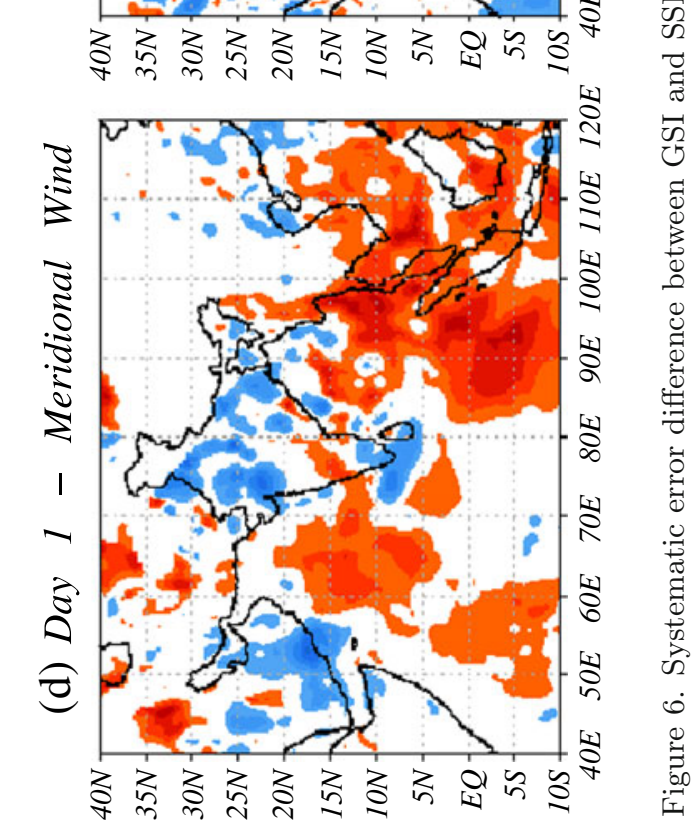

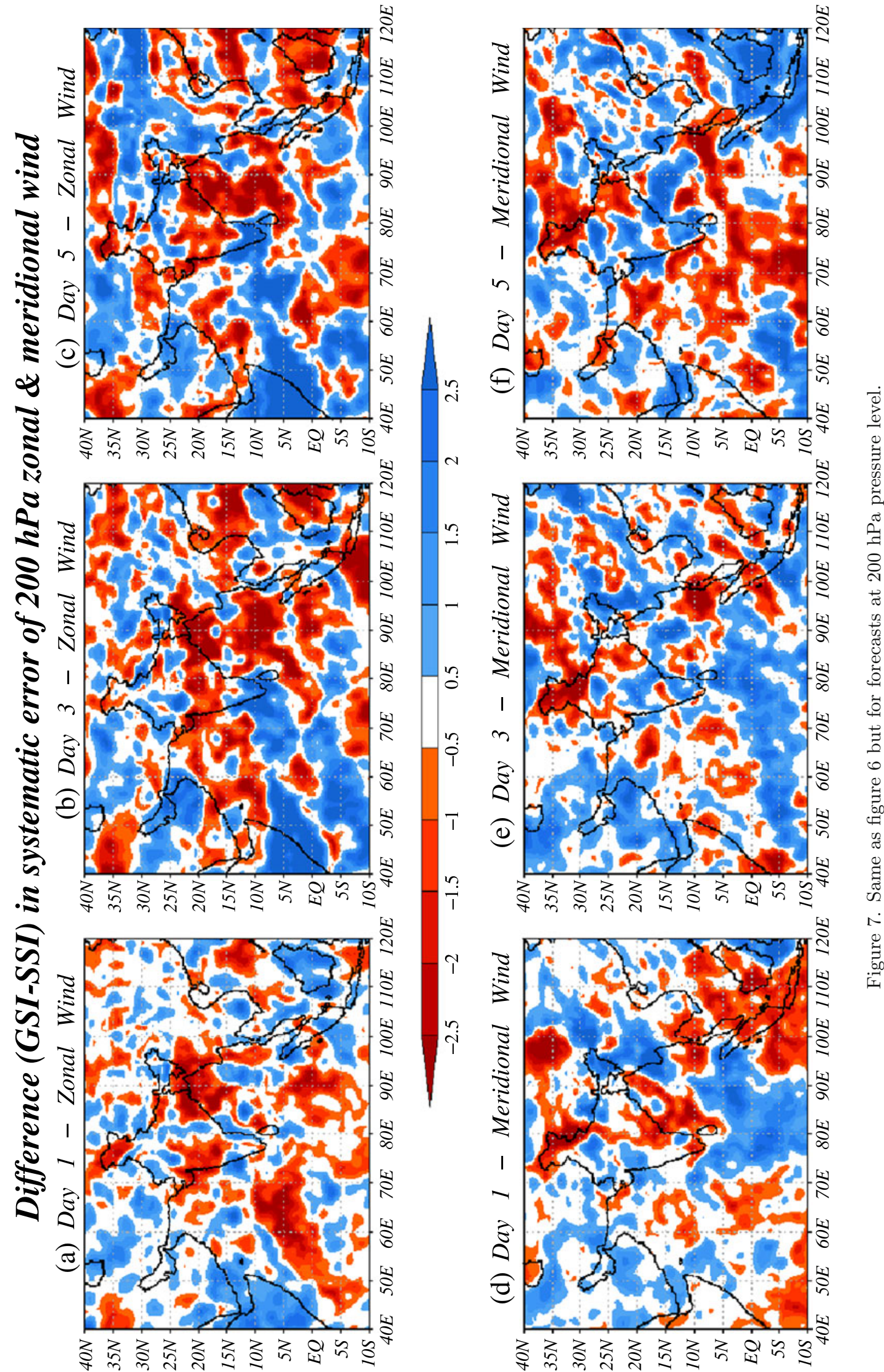

₹

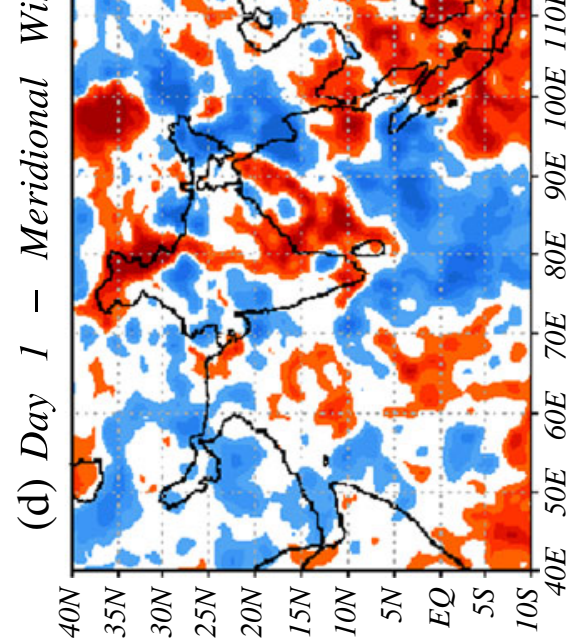


in the month of June 2008, depressions formed over east central Arabian Sea (5-7 June 2008) and north Bay of Bengal (16-18 June 2008) were significant. On 17 June, 2008 the depression formed over the north Bay of Bengal, lay over gangetic West Bengal bringing heavy rainfall over the region. On this day, West Bengal received heavy rainfall of about $115-120 \mathrm{~mm}$, highest for the month over the state. So for the present study, this day was selected as one of the day for rainfall comparisons between the two simulations. The depression over the north Bay of Bengal is selected as a case study of depression. The depression and the rainfall comparison have been discussed in detail below. The above-stated information for June 2008 were taken from Climate Diagnostics Bulletin of India (June 2008) and Mazumdar et al (2009).

\subsubsection{Depression}

For the study of depression, the north Bay of Bengal case has been selected. It had longer life than the Arabian Sea case and had crossed the coast and entered inland. It lay over gangetic West Bengal and adjoining Bangladesh, centered close to lat. $23.0^{\circ} \mathrm{N}$ and long. $88.5^{\circ} \mathrm{E}$, about $80 \mathrm{~km}$ northnortheast of Kolkata, at 0300 UTC of 17 June and over gangetic West Bengal, close to lat. $23.0^{\circ} \mathrm{N}$ and long. $88.0^{\circ} \mathrm{E}$, about $100 \mathrm{~km}$ northwest of Kolkata at 1200 UTC. Figures 8 and 9 depict the $925 \mathrm{hPa}$

\section{$925 \mathrm{hPa}$ horizontal wind $(\mathrm{m} / \mathrm{s})$ and geo-potential (gpm) GSI - 00UTC 17th June, 2008}

(a)

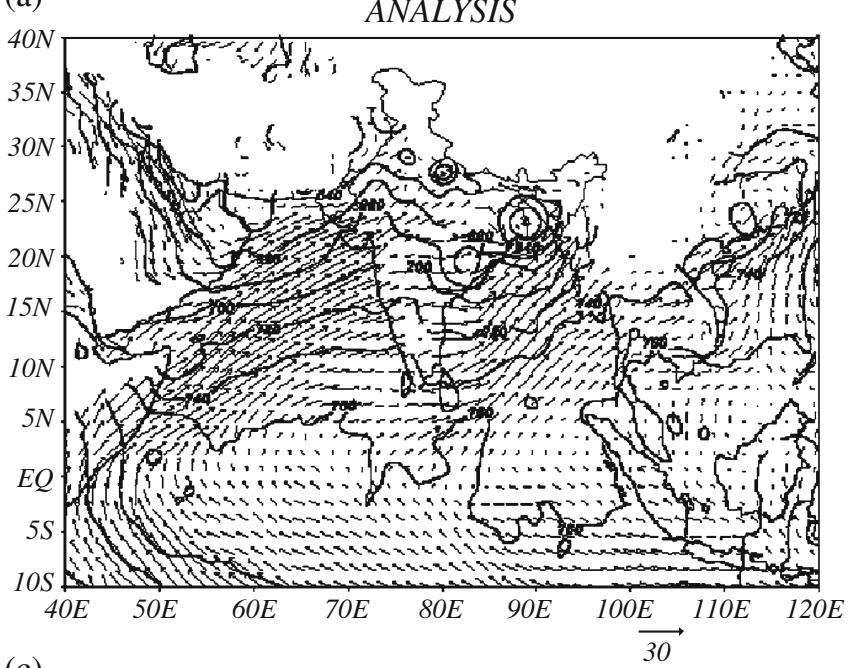

(c)

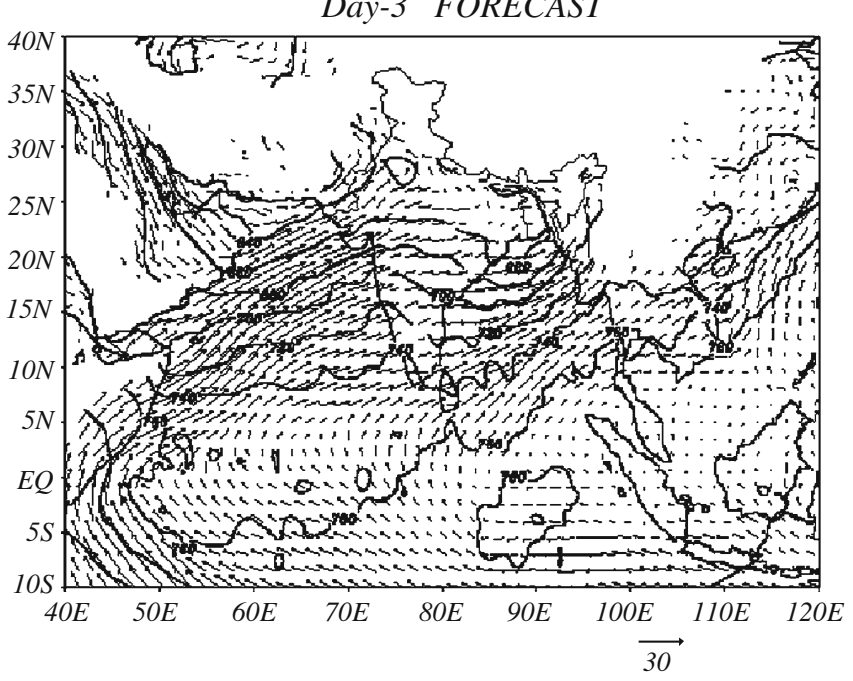

(b)

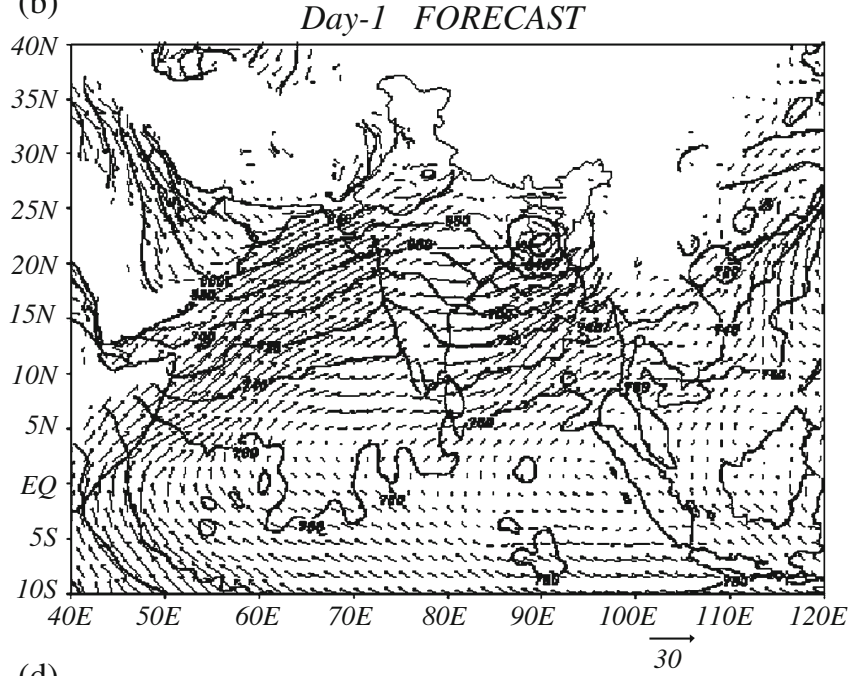

(d)

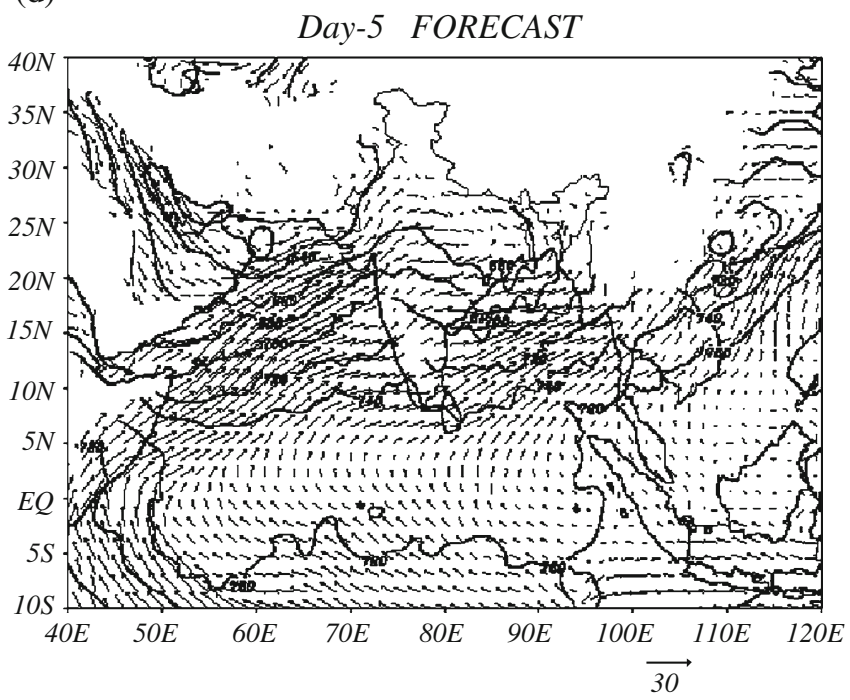

Figure 8. $925 \mathrm{hPa}$ horizontal wind $(\mathrm{m} / \mathrm{s})$ and geopotential (gpm) at 00UTC of 17 June 2008 for GSI (a) analysis and the corresponding (b) day-1, (c) day-3, and (d) day-5 forecasts. 
horizontal wind $(\mathrm{m} / \mathrm{s})$ and geopotential (gpm) for GSI and SSI analyses (figures 8a and 9a) and their corresponding day-1 (figures $8 \mathrm{~b}$ and $9 \mathrm{~b}$ ), day-3 (figures $8 \mathrm{c}$ and $9 \mathrm{c}$ ) and day-5 (figures $8 \mathrm{~d}$ and 9d) forecasts valid at 00UTC of 17 June, 2008, respectively. It is seen that the depression in the GSI analysis is more intense than that in SSI. In GSI analysis the depression is centered close to the observation position whereas SSI has shifted it little southwards. GSI has captured the system in its day-1 forecast but was unable to produce it in day3 and day-5 forecasts. On the contrary, SSI has been able to produce the system in both day-1 and day-3 forecasts and weakened the same in day- 5 forecasts. The forecast centre of the depression in both GSI and SSI is southwards of its position in the analysis.

\subsubsection{Rainfall}

Day-1, day-3 and day-5 rainfall forecasts valid at same day are compared with that of TRMM3B42V6 accumulated daily rainfall. TRMM rainfall is shown in figure 10. Figure 11 depicts the rainfall forecasts of GSI (figure $11 \mathrm{a}, \mathrm{b}$ and $\mathrm{c}$ ) and SSI (figure 11d, e and f) valid at 00z of 17 June, 2008. Both GSI and SSI simulated outputs overestimate the rainfall amount especially along the western coast of India. Heavy rainfall event over coastal West Bengal is better captured by day-1 SSI forecast valid at $00 \mathrm{z}$ of 17 June, 2008. In day-3 and day- 5 forecasts both GSI and SSI are unable to produce the heavy downpour over the coasts of West Bengal. With advance of forecast days, SSI shows an increased overestimation of rainfall over central

\section{$925 \mathrm{hPa}$ horizontal wind ( $\mathrm{m} / \mathrm{s})$ and geo-potential (gpm)}

SSI - 00UTC 17th June, 2008

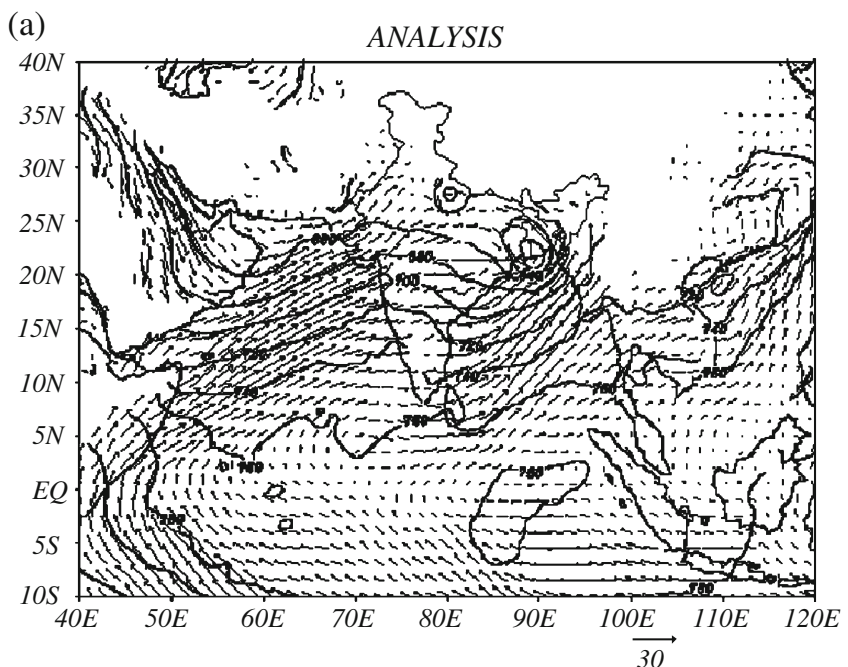

(c)

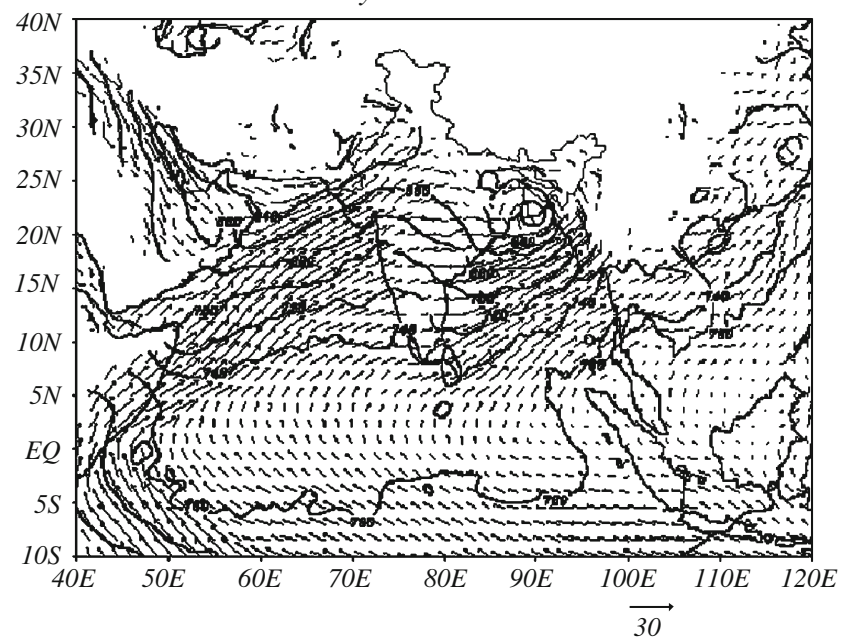

(b)

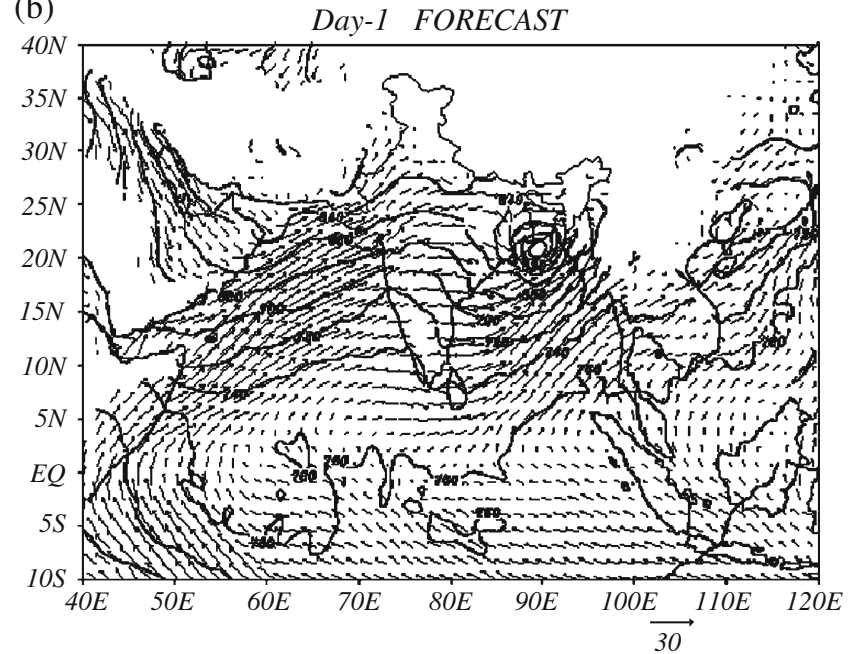

(d)

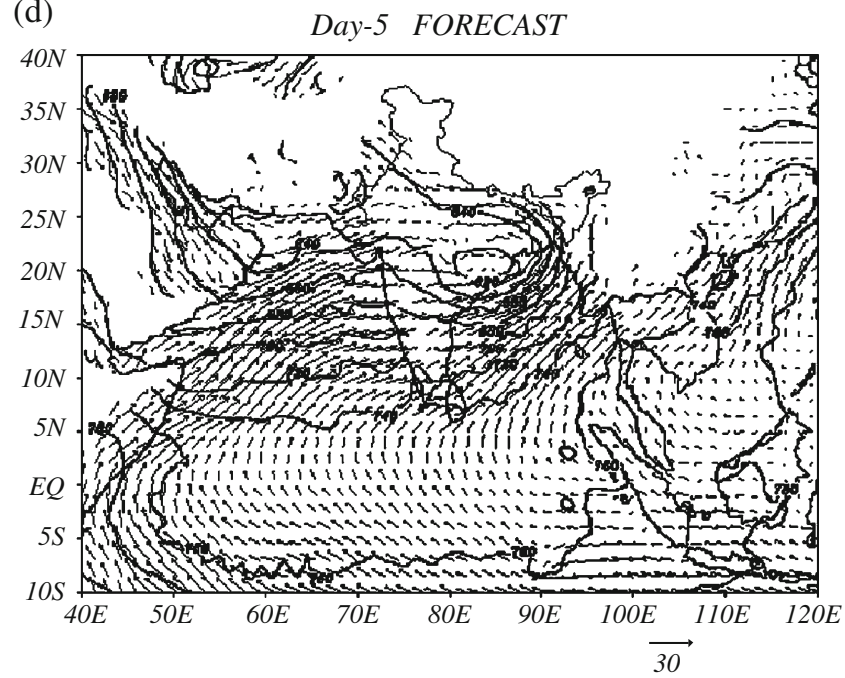

Figure 9. Same as figure 8 but for SSI. 
and south-eastern India. It is found that rainfall forecast of SSI is better at day-1 whereas GSI forecasts over central, north-central and eastern India is closer to the observation for day- 5 forecasts valid at the same day.

An objective method for predicting monsoon onset, advancement and withdrawal date using dynamic and thermodynamic precursors from NCMRWF T80L18 analysis-forecast system was developed (Ramesh et al 1996; Basu et al 1999) and used since 1995 at NCMRWF. The same methodology has been extended for this study for comparing the performance of GSI and SSI. Two regions, one in Bay of Bengal $\left(0^{\circ}-19.5^{\circ} \mathrm{N}, 78^{\circ}-96^{\circ} \mathrm{E}\right)$ and the other in Arabian Sea $\left(0^{\circ}-19.5^{\circ} \mathrm{N}, 55.5^{\circ}-75^{\circ} \mathrm{E}\right)$ were selected. Daily variation of the $850 \mathrm{hPa}$ kinetic energy, mean tropospheric temperature (MTT) and net tropospheric moisture (NTM) from the analyses and forecast fields were calculated. Figures 12 and 13 depict these values as produced by GSI and SSI analyses over Bay of Bengal and Arabian Sea, respectively. The pattern of daily variation of all the three parameters is similar for both the analyses. Peak in $850 \mathrm{hPa}$ kinetic energy and MTT and corresponding decrease of NTM shows active rainfall episodes over the region. The values of SSI analyses are mostly found to be higher than those produced by GSI analyses scheme. The results were similar for the forecast days (figures not included) with values from SSI scheme higher than the GSI scheme.

\section{Summary}

From the present study, it is clearly seen that over tropics, correlation of temperature anomaly and pattern of GSI forecast with respect to its analysis are higher compared to SSI at both lower and upper layers. For wind, GSI has higher anomaly and pattern correlation at lower layers whereas at upper layers the results are mixed. Vector wind RMSE from GSI forecasts were lower to that of SSI over both upper and lower levels. But over Indian region, forecasts from GSI have lower vector wind RMSE at lower level and higher RMSE at upper level. For the temperature field over Indian region, forecasts from GSI have higher correlation with its analysis and lower RMSE both at upper and lower levels. Improvement in systematic errors of GSI simulated temperature at upper and lower levels is observed whereas SSI has mostly lower wind error. Improvement in systematic errors by GSI is mostly found over the peninsular and southern coast of

TRMM 3B42V6 Daily Rainfall VALID ON 00Z17JUN2008 RAINFALL $(\mathrm{cm}) \mathrm{CI}=0.1,1,2,4,8, .$.

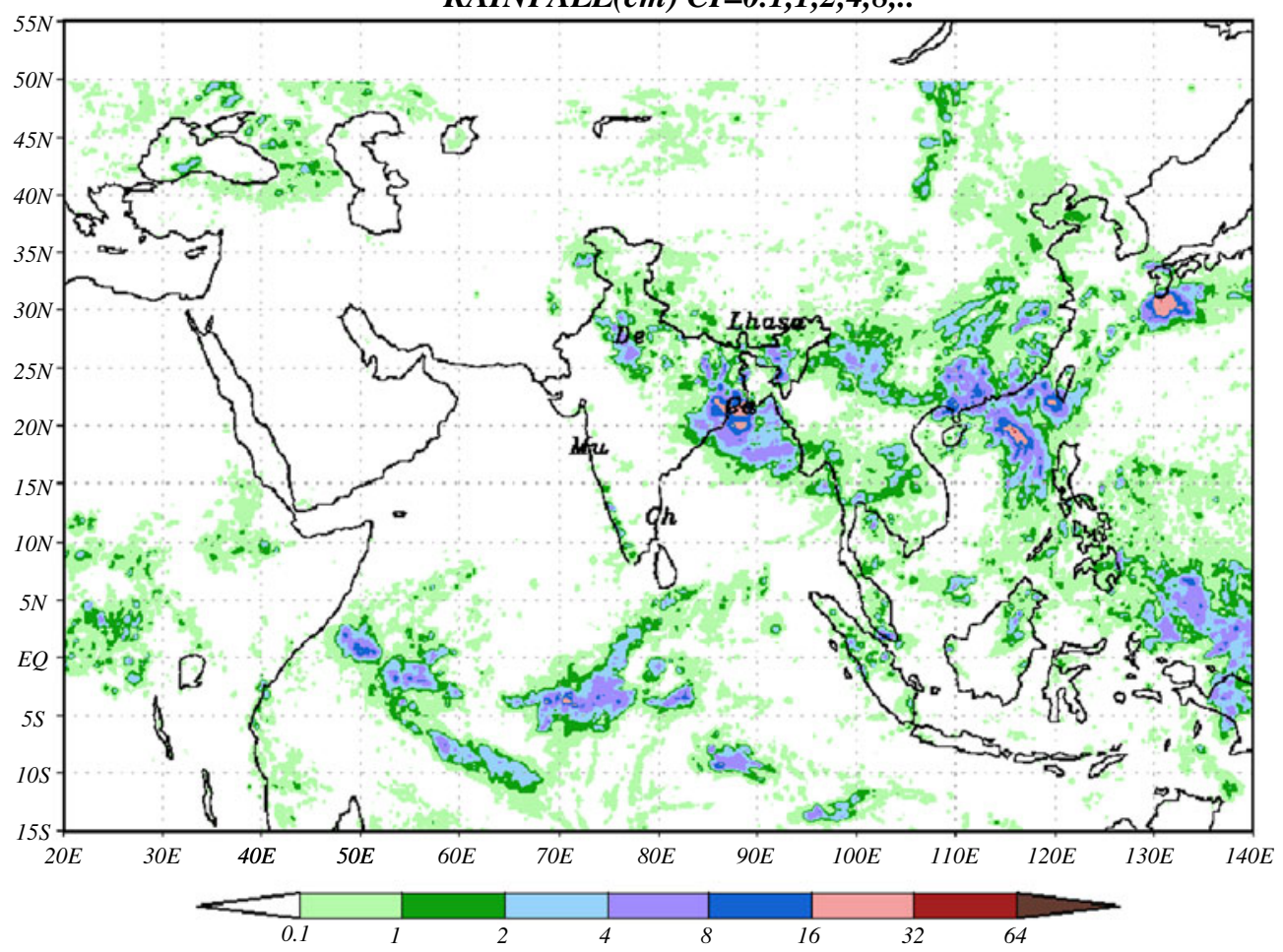

Figure 10. TRMM 3B42-V6 accumulated rainfall (mm) valid at 00z of 17 June, 2008. 

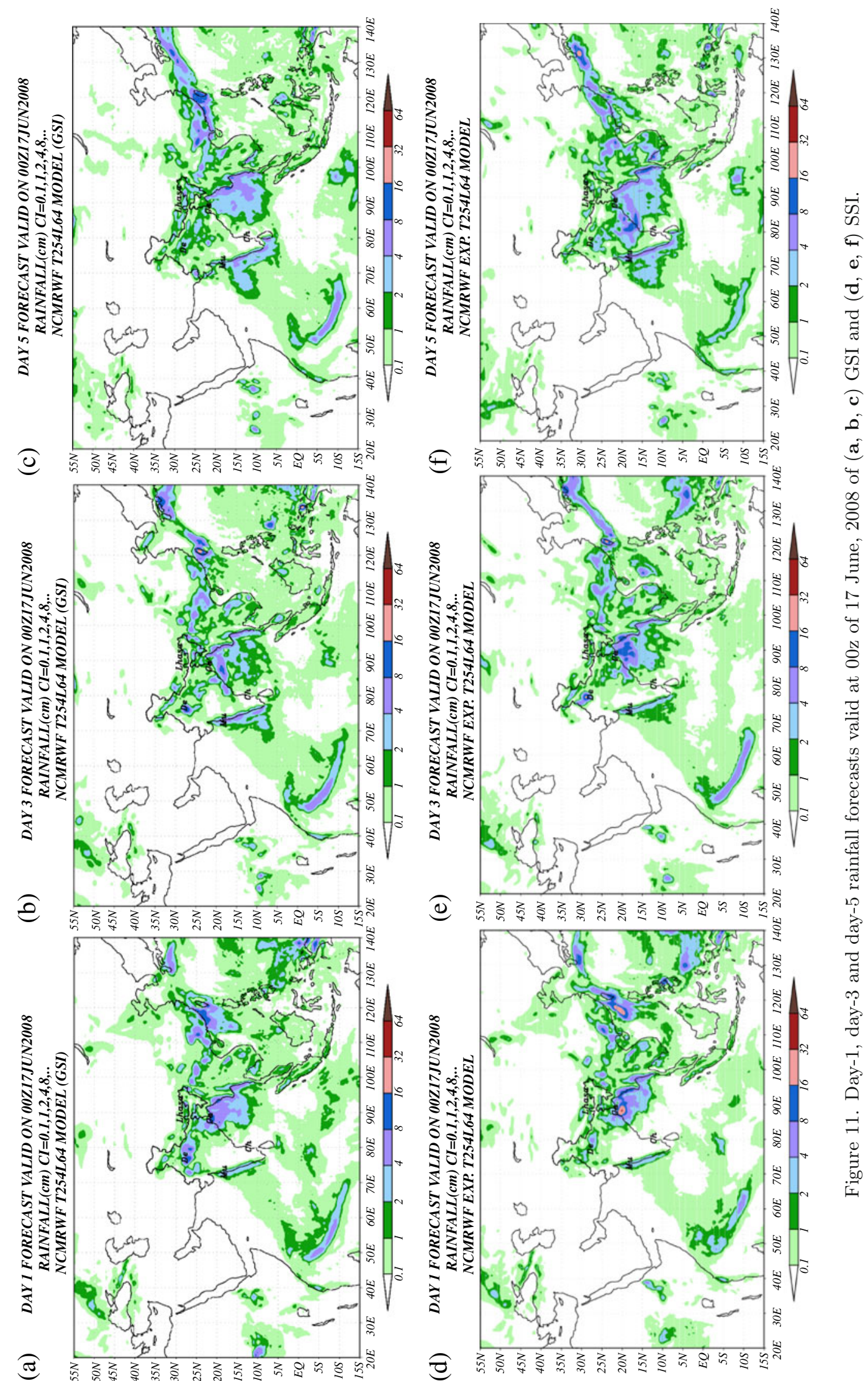


\section{Daily Variation over Bay of Bengal}

(a) 850hPa Kinetic Energy (Joules)

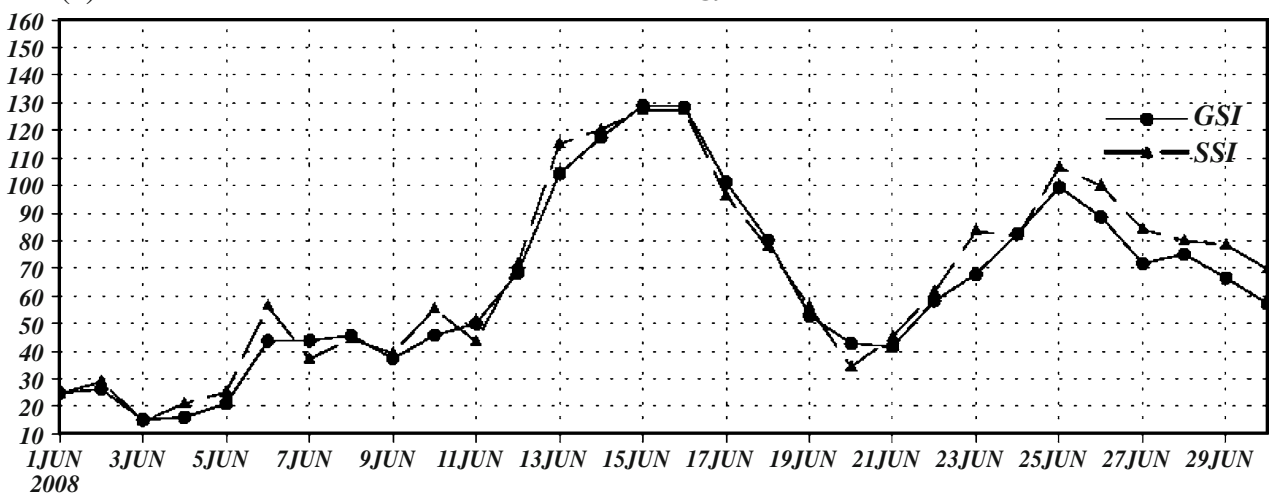

(b) Mean Tropospheric Temperature (Kelvin)

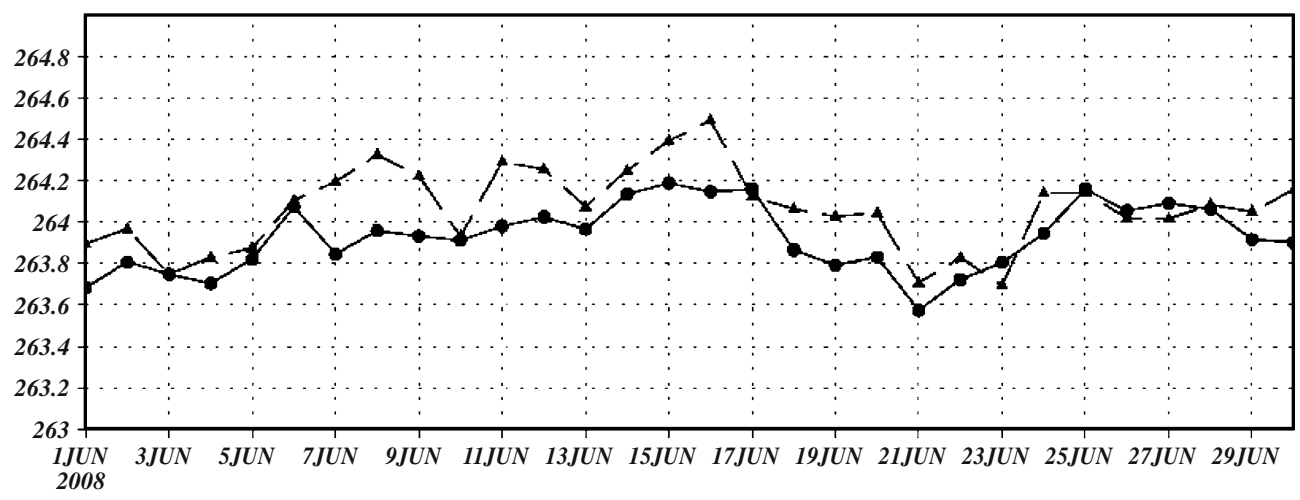

(c)

Net Tropospheric Moisture (mm)

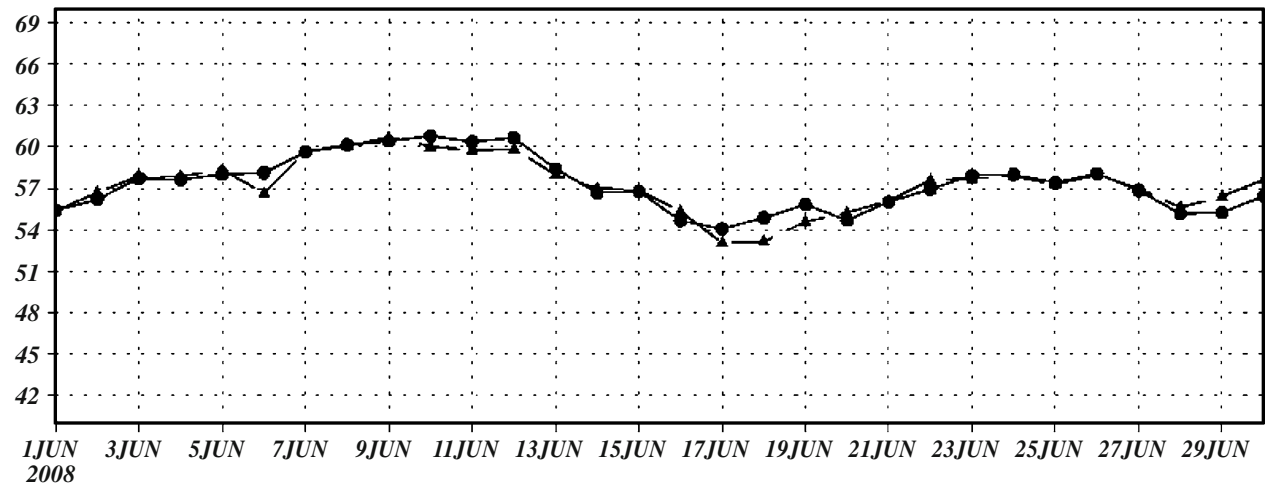

Figure 12. Daily variation of (a) $850 \mathrm{hPa}$ kinetic energy (joules), (b) mean tropospheric temperature (kelvin) and (c) net tropospheric moisture $(\mathrm{mm})$ as produced by the GSI and SSI analyses over Bay of Bengal $\left(0^{\circ}-19.5^{\circ} \mathrm{N}, 78^{\circ}-96^{\circ} \mathrm{E}\right)$ region.

India. When model forecasts were compared with the observations, consistent improvement in GSI simulated temperature forecasts at upper level was observed. From the synoptic case study, it was found that the depression centre in GSI analysis is closer to observation but it has produced more intense depression compared to that of SSI. Consistent improvement in rainfall forecasts was not observed. Also, the daily values of $850 \mathrm{hPa}$ mean kinetic energy, MTT and NTM from SSI analyses scheme is higher than the values obtained from GSI.

From the current study, it is evident that GSI analysis scheme do have positive impact on most of the fields over tropics and India and its surrounding regions. Though not for all but for some fields it is in edge over spectral statistical analysis scheme. Improvement in model analyses by GSI over the 


\section{Daily Variation over Arabian Sea}

(a) 850hPa Kinetic Energy (Joules)

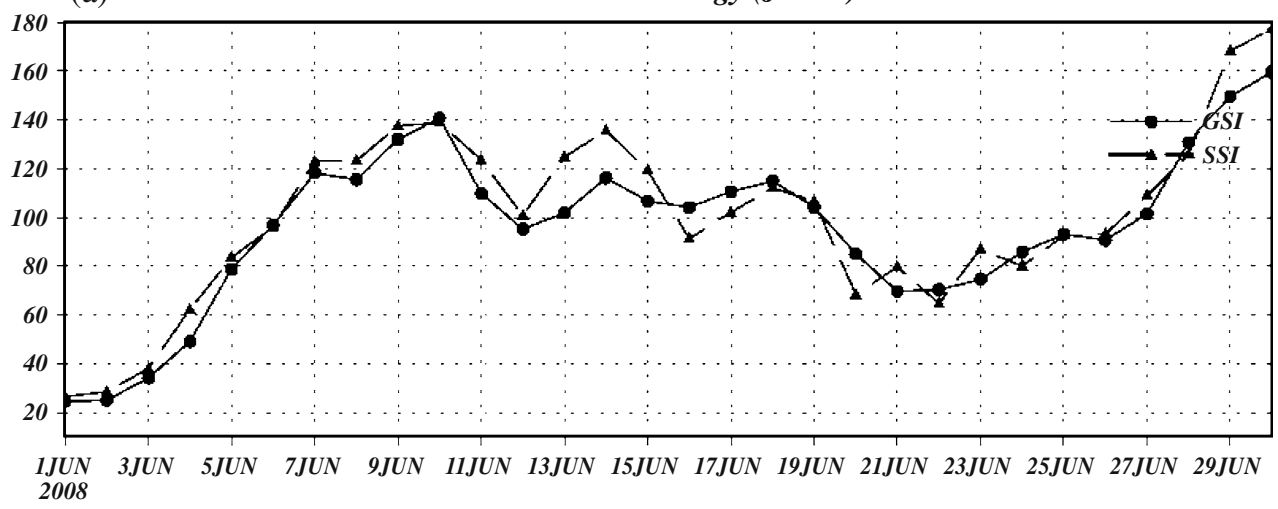

(b)

Mean Tropospheric Temperature (Kelvin)

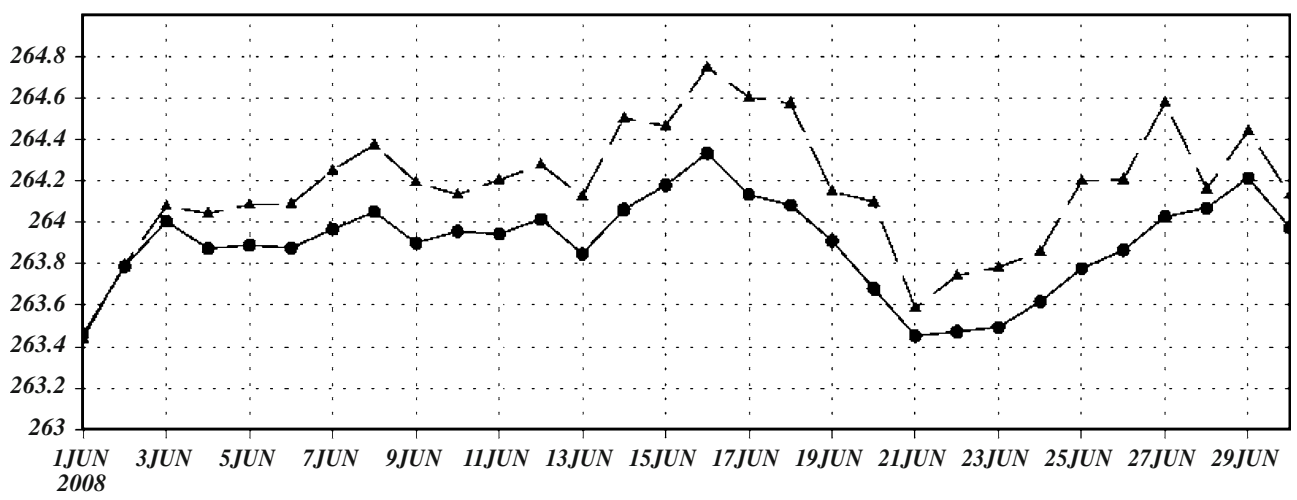

(c)

Net Tropospheric Moisture (mm)

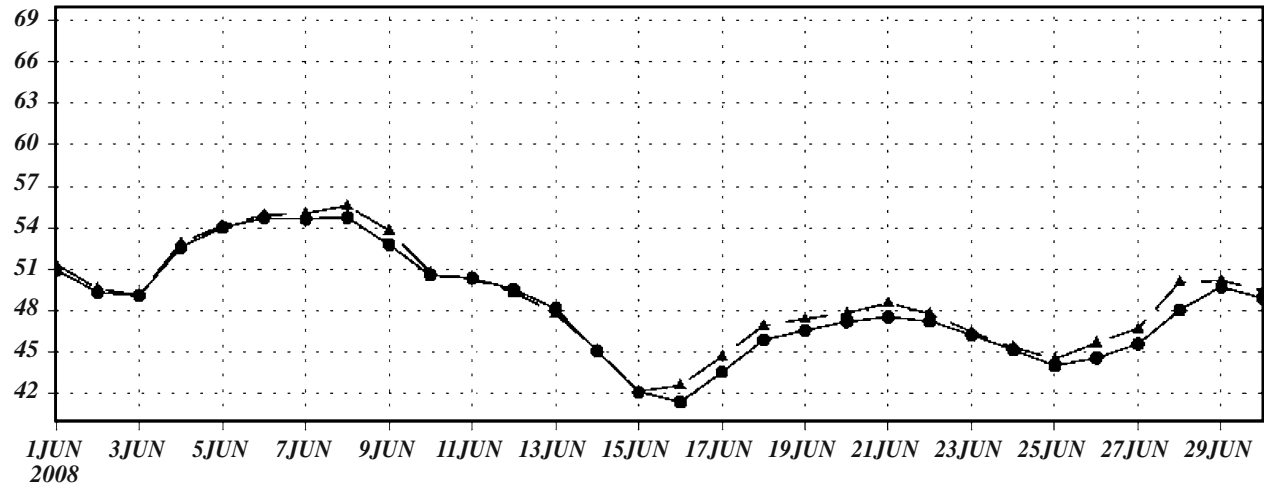

Figure 13. Same as figure 12 but over Arabian Sea $\left(0^{\circ}-19.5^{\circ} \mathrm{N}, 55.5^{\circ}-75^{\circ} \mathrm{E}\right)$ region.

tropical region has been established. As from this study overall improvement of GSI above SSI cannot be stated; work on upgradation of GSI is continuing to overcome its flaws and demerits compared to SSI analysis system.

\section{Acknowledgements}

We gratefully acknowledge National Centre for Environmental Prediction (NCEP) for sharing the GSI code. The first author gratefully acknowledges Council of Scientific and Industrial Research (CSIR) for providing the research fellowship. We thank Dr E N Rajagopal for his guidance and help to carry out this research work. We thank Dr A K Bohra (Former Head, NCMRWF) for providing the necessary facilities to carry out this research work and for his encouragement and support. We would like to thank the anonymous referees for their valuable comments which improved the quality of the manuscript. 


\section{References}

Basu S, Ramesh K J and Begum Z N 1999 Medium range prediction of summer monsoon activities over India vis-àvis their correspondence with observational features; $A d v$. Atmos. Sci. 16(1) 133-146.

Climate Diagnostics Bulletin of India 2008 Government of India, India Meteorological Department.

Courtier P and Coauthors 1998 The ECMWF implementation of the three-dimensional variational assimilation (3D-Var). I: Formulation; Quart. J. Roy. Meteorol. Soc. 124 1783-1807.

Mazumdar A B, Khole M and Sunitha Devi S 2009 Weather in India. Monsoon Season (June to September, 2008); Mausam 60(3) 379-426.

Pailleux J 1990 A global variational assimilation scheme and its application for using TOVS radiances; Preprints, WMO International Symposium on Assimilation of Observations in Meteorology and Oceanography, ClermontFerrand, France, WMO, pp. 325-328.

Parrish D F and Derber J C 1992 The National Meteorological Center's Spectral Statistical-Interpolation Analysis System; Mon. Weather Rev. 120 1747-1763.
Purser R J, Wu W-S, Parrish D F and Roberts N M 2003a Numerical aspects of the application of recursive filters to variational statistical analysis. Part I: Spatially homogeneous and isotropic gaussian covariances; Mon. Weather Rev. 131 1524-1535.

Purser R J, Wu W-S, Parrish D F and Roberts N M 2003b Numerical aspects of the application of recursive filters to variational statistical analysis. Part II: Spatially inhomogeneous and anisotropic general covariances; Mon. Weather Rev. 131 1536-1548.

Rajagopal E N, Das Gupta M, Mohandas S, Prasad V S, George J P, Iyengar G R and Preveen Kumar D 2007 Implementation of T254L64 global forecast system at NCMRWF; NCMRWF Technical Report, pp. 1-42.

Ramesh K J, Basu S and Begum Z N 1996 Objective determination of onset, advancement and withdrawal of the summer monsoon using large scale forecast fields of a global spectral model over India; Meteorol. Atmos. Phys. 61 137-151.

Wu W-S, Purser R J and Parrish D F 2002 Threedimensional variational analysis with spatially inhomogenous covariances; Mon. Weather Rev. 130 2905-2916. 